\title{
Cloud Shading Effects on Characteristic Boundary-Layer Length Scales
}

\author{
G. L. Horn ${ }^{1}$ - H. G. Ouwersloot ${ }^{2}$. \\ J. Vilà-Guerau de Arellano ${ }^{1}$ - M. Sikma ${ }^{1}$
}

Received: 14 August 2014 / Accepted: 18 June 2015 / Published online: 17 July 2015

(C) The Author(s) 2015. This article is published with open access at Springerlink.com

\begin{abstract}
We studied the effects of shading by shallow cumulus (shallow $\mathrm{Cu}$ ) and the subsequent effect of inducing heterogeneous conditions at the surface on boundary-layer characteristics. We placed special emphasis on quantifying the changes in the characteristic length and time scales associated with thermals, shallow $\mathrm{Cu}$ and induced thermal circulation structures. A series of systematic numerical experiments, inspired by Amazonian thermodynamic conditions, was performed using a large-eddy simulation model coupled to a land-surface model. We used four different experiments to disentangle the effects of shallow $\mathrm{Cu}$ on the surface and the response of clouds to these surface changes. The experiments include a 'clear case', 'transparent clouds', 'shading clouds' and a case with a prescribed uniform domain and reduced surface heat flux. We also performed a sensitivity study on the effect of introducing a weak background flow. Length and time scales were calculated using autocorrelation and two-dimensional spectral analysis, and we found that shading controlled by shallow $\mathrm{Cu}$ locally lowers surface temperatures and consequently reduces the sensible and latent heat fluxes, thus inducing spatial and temporal variability in these fluxes. The length scale of this surface heterogeneity is not sufficiently large to generate circulations that are superimposed on the boundary-layer scale, but the heterogeneity does disturb boundary-layer dynamics and generates a flow opposite to the normal thermal circulation. Besides this effect, shallow $\mathrm{Cu}$ shading reduces turbulent kinetic energy and lowers the convective velocity scale, thus reducing the mass flux. This hampers the thermal lifetime, resulting in a decrease in the shallow $\mathrm{Cu}$ residence time (from 11 to $7 \mathrm{~min}$ ). This reduction in lifetime, combined with a decrease in mass flux, leads to smaller clouds. This is partially compensated for by a decrease in thermal cell size due to a reduction in turbulent kinetic energy. As a result, inter-cloud distance is reduced, leading to a larger population of smaller clouds, while maintaining cloud
\end{abstract}

G. L. Horn

geertenhorn@gmail.com

J. Vilà-Guerau de Arellano jordi.vila@wur.nl

1 Meteorology and Air Quality Section, Wageningen University, Wageningen, The Netherlands

2 Max Planck Institute for Chemistry, Mainz, Germany 
cover similar to the non-shading clouds experiment. Introducing a $1 \mathrm{~m} \mathrm{~s}^{-1}$ background wind speed increases the thermal size in the sub-cloud layer, but the diagnosed surface-cloud coupling, quantified by characteristic time and length scales, remains.

Keywords Atmospheric length scales - Boundary-layer length scales · Cloud size · Dynamic heterogeneity · Large-eddy simulation · Shallow cumulus shading · Surface-cloud coupling

\section{Introduction}

Land-atmosphere feedback loops are a major source of uncertainty in current climate models even though they play an important role in climate simulations (Bonan 1995; Stephens 2005; Seneviratne et al. 2006; Neggers et al. 2007). One of the most important feedbacks within the land-atmosphere system concerns the influence of clouds (Tiedtke et al. 1988; Huang and Margulis 2013). This uncertainty increases with shallow cumulus (shallow $\mathrm{Cu}$ ) because of the strong dependence of this cloud type on surface conditions, their chaotic behaviour and their short time scales. Shallow $\mathrm{Cu}$ can be the first stage of deep convection (Kuang and Bretherton 2006), that is a key factor in convective weather systems (Xu and Zipser 2012). A better understanding of shallow $\mathrm{Cu}$ interactions with the surface is therefore of importance for climate research and weather prediction.

One of the land-atmosphere feedback loops that still requires attention is related to the interaction between surface characteristics, shallow $\mathrm{Cu}$ and the heterogeneities associated with this coupling. It is important to stress that here we distinguish between two sorts of surface heterogeneities in which shallow $\mathrm{Cu}$ plays a relevant role: dynamic heterogeneity and static heterogeneity. Heterogeneities that are created by non-uniform surface conditions (e.g. differences in roughness, soil moisture or plant type) are regarded as static heterogeneities. Dynamic heterogeneities are spatial non-uniformities of surface variables created by self-organization of turbulence and land-atmosphere feedbacks (e.g. shallow $\mathrm{Cu}$ shading, subsidence and local evaporation) over an initially uniform surface.

Here we focus on the effects of dynamic heterogeneity produced by shallow $\mathrm{Cu}$ shading, on atmospheric boundary-layer (ABL) characteristics, with emphasis on length scales related to thermals and circulation structures. In contrast to dynamic heterogeneities, there has been much written on the effects of (static) heterogeneity in surface fluxes on cloud formation (Avissar and Liu 1996; Golaz et al. 2001; van Heerwaarden and Vilà-Guerau de Arellano 2008), while other studies (e.g. Avissar and Schmidt 1998; Baidya Roy and Avissar 2000; Patton et al. 2005) focussed on characteristics in a (static) heterogeneously forced ABL.

Avissar and Schmidt (1998) and Patton et al. (2005) focussed on length scales associated with secondary circulations induced by static heterogeneity. They suggest that heterogeneities with length scales of five to eight times the ABL height and 5-10 km induce circulations that have the largest effect on the ABL. Ouwersloot et al. (2011) showed that heterogeneities with length scales between 2 and 16 times the ABL height affect the structure of the ABL.

Dynamic heterogeneity is a topic that has been addressed in the field of smoke aerosols (e.g. Jiang and Feingold 2006; Jiang et al. 2009). Using a coupled land-surface large-eddy simulation (LES) model, Jiang and Feingold (2006) showed that, if smoke aerosols act as cloud condensation nuclei (CCN), they can lead to an increase in cloud optical depth through microphysical modification, which is countered by a decrease in cloud optical depth as a result of aerosol radiative effects. As a result of aerosol effects on radiative scattering, surface fluxes decrease, which in turn leads to weaker convection, lower cloud optical depths, lower aerosol 
optical depths and a lower cloud fraction. Also in the field of aerosols, Xue and Feingold (2006) showed the development of smaller clouds when the aerosol load increases, and also discussed the possibility of shorter cloud lifetimes in cases in which more aerosols are present.

In contrast, few studies have quantified the interactive coupling between shallow $\mathrm{Cu}$ and the land-surface characteristics connected to cloud shading (dynamic heterogeneity). Lohou and Patton (2014) showed that clouds can introduce dynamic heterogeneity by shading the surface, that dynamic heterogeneity influences the energy balance due to reduced incoming solar radiation in shaded areas and that turbulence and secondary circulations, created by the variability in surface properties, increase the spatial variability of surface fluxes. Their study demonstrated that clouds enhance entrainment rates and therefore locally dry the ABL, causing a higher spatial variability in moisture content. As an effect closely connected with the drying effect, they showed that the presence of shallow $\mathrm{Cu}$ increases the evaporative fraction and spatial surface-flux variability. This leads to cloud-induced surface heterogeneities, which are less than $1 \mathrm{~km}$ wide on the horizontal scale, influencing the vertical fluxes of moisture and heat. Vilà-Guerau de Arellano et al. (2014) showed that shallow $\mathrm{Cu}$ shading leads to a high spatial variability in the surface fluxes and that a coupling between clouds and the surface leads to less extreme values of liquid water path (LWP). To the best of our knowledge, Jiang and Feingold (2006), Vilà-Guerau de Arellano et al. (2014) and Lohou and Patton (2014) are the only numerical studies of shallow $\mathrm{Cu}$ in which an interactive land surface is employed to explicitly take into account coupling between the land surface and shallow $\mathrm{Cu}$.

The above-mentioned studies on static heterogeneity have shown that the length scale of the heterogeneity is an important characteristic describing the dynamics of the atmospheric flow. Lohou and Patton (2014) briefly addressed length scales associated with cloud-induced dynamic heterogeneity and related circulations. The novelty of our study is that it addresses these ABL length scales of thermal-induced circulations associated with the cloud-induced heterogeneities. We take this cloud effect on surface fluxes into account by performing systematic experiments, by running a LES model coupled to an interactive land-surface model (LSM). For this we choose the Dutch atmospheric LES model (DALES; Heus et al. 2010); a numerical one is chosen because it is impossible to determine these characteristics from experimental data.

Due to the experimental design, we analyze and compare cases with: (i) shallow $\mathrm{Cu}$ shading the surface, (ii) clouds transparent to radiation that therefore do not shade, (iii) a clear ABL with identical initial conditions to those of the other experiments but where condensation is disabled and (iv) a case similar to (i) but with prescribed surface fluxes similar to the domain average of (i). This gives us the capacity to break down the complexity of the atmospheric flow and distinguish between the different effects of clouds on ABL characteristics, concentrating on the thermals and circulations. We analyze in detail length scales based on both cloud optical depth $(\tau)$ and vertical wind velocity $(w)$ associated with cloud development and thermal circulation, respectively. Previous work on length scales of vertical wind velocity was mainly based on clear cases without a cloud-surface coupling (Lenschow and Stankov 1986; De Roode et al. 2004; Dosio et al. 2005; Pino et al. 2006; Verzijlbergh et al. 2009; Hellsten and Zilitinkevich 2013). This coupling is taken into account in this study.

The length scales of interest, further explained in Sect. 2.3, are the horizontal length scales of the circulation structures, the horizontal length scales of the clouds that are closely related to the length scales of the thermals, and the distance between clouds. Changes in the length scales of the vertical velocity between the case with shading clouds and the other model set-ups could indicate that the thermally induced circulations are affected by shading and 
therefore dynamic heterogeneity. The length and time scales associated with these motions are determined by using autocorrelation analysis (Lenschow and Stankov 1986; Dosio et al. 2005; Verzijlbergh et al. 2009) and spectral analysis (e.g. Lenschow and Stankov 1986; De Roode et al. 2004; Pino et al. 2006; Hellsten and Zilitinkevich 2013). These methods are less computationally intensive than for example the also recently employed cloud tracking (e.g. Jiang et al. 2006; Dawe and Austin 2012; Heus et al. 2013) but are still accurate in their prediction of the length and time scales.

The paper is structured as follows: Sect. 2 describes our methodology, including the numerical set-up of the LES model, the systematic experiments and the techniques to calculate the length and time scales. The simulation results are presented and discussed in Sect. 3. Section 4 includes a discussion, showing the main relations between the different length scales and the effect of clouds, with our conclusions and recommendations set out in Sect. 5.

\section{Methods}

\subsection{Numerical Model}

The numerical experiments were performed with the fourth version of the DALES model ${ }^{1}$ based on version 3.2 (Heus et al. 2010). The code has been optimized and contains some new elements compared with version 3.2. Ninety per cent of the turbulent energy is explicitly resolved (Heus et al. 2010), while the subgrid scale is solved with the sub-filter-scale turbulent kinetic energy (TKE) model (SFS-TKE model) (Deardorff 1980). The DALES model employs the Boussinesq approximation to solve the Navier-Stokes equations using a thirdorder Runge-Kutta scheme with a no-slip boundary condition at the bottom and periodic boundary conditions at the sides to create an infinite repetitive domain eliminating lateral boundary effects. At the top one-third of the grid, a sponge layer is introduced to dampen fluctuations that can be introduced due to the reflection of gravity waves.

The DALES model is coupled to the surface by parameterizing the surface scalar fluxes and surface momentum fluxes with a land-surface model (LSM) (Heus et al. 2010). The LSM consists of two components: one to resolve the local surface energy balance, and the other to resolve a four-layer soil scheme. The coupling between the DALES model and the LSM has been discussed by van Heerwaarden (2011).

Here, cloud formation in the DALES model is based on condensation only, on the assumption that all water above saturation condenses (Heus et al. 2010). This scheme was previously used by Siebesma et al. (2003) and van Stratum (2011) to successfully represent cumulus over land. Siebesma and Jonker (2000) showed that the DALES model is capable of reproducing the macroscale nature of clouds, indicating the ability to perform a cloud study. In one of our simulations, this calculation of liquid water is skipped to prevent cloud formation. No precipitation scheme is implemented, which is a simplification compared with typical Amazonian conditions, but is desirable in order to isolate the effects of cloud shading. The radiative scheme employed is one-dimensional (1D). The sun is thus always located overhead during the day, and clouds always shade the surface directly below. This is a valid approximation for daytime conditions around the Equator, where the sun is almost directly overhead during the most important time for cloud formation. It is important to note that the radiation intensity still follows the diurnal cycle.

${ }^{1}$ Version 4.0 of the DALES model is not yet published, but the code is available. 


\subsection{Design of Numerical Experiments}

The numerical experiments were designed to reproduce shallow $\mathrm{Cu}$ over a homogeneous surface under Amazonian conditions and the effects of shallow $\mathrm{Cu}$ shading. A domain of $24 \mathrm{~km} \times 24 \mathrm{~km} \times 6 \mathrm{~km}$ is employed, with grid spacings of $50 \mathrm{~m} \times 50 \mathrm{~m} \times 20 \mathrm{~m}$. Sullivan et al. (1998) and van Heerwaarden (2006) indicate that, at this resolution, most of the TKE lies in the resolved scale.

The initial atmospheric conditions used are based on the TROpical Forest and Fire Emissions Experiment (TROFFEE) (Karl et al. 2007; Vilà-Guerau de Arellano et al. 2011), adapted by Ouwersloot et al. (2013) to ensure that shallow Cu convection occurs with no deep convection. The initial conditions follow Amazonian surface and upper-air conditions: the initial boundary-layer height is $200 \mathrm{~m}$ with a potential temperature $(\theta)$ of $300 \mathrm{~K}$ and a specific humidity $(q)$ of $16 \times 10^{-6} \mathrm{~kg} \mathrm{~kg}^{-1}$. The initial jumps from the mixed layer to the free troposphere for $\theta(\Delta \theta)$ and $q(\Delta q)$ are $1 \mathrm{~K}$ and $-3 \times 10^{-6} \mathrm{~kg} \mathrm{~kg}^{-1}$, respectively. In accordance with Brown (2002), the tropospheric $\theta$ gradient is $3 \times 10^{-3} \mathrm{~K} \mathrm{~m}^{-1}$ up to $4 \mathrm{~km}$ and $6 \times 10^{-3} \mathrm{~K} \mathrm{~m}^{-1}$ above $4 \mathrm{~km}$ (Ouwersloot et al. 2013). The specific humidity gradient in the troposphere is $-3 \times 10^{-6} \mathrm{~m}^{-1}$ until the specific humidity becomes zero. The incoming radiation is based on the location (latitude $2.612^{\circ} \mathrm{S}$, longitude $60.910^{\circ} \mathrm{W}$ ), day of year (263) and time of day. We consider cases for zero wind speed because advection influences the strength of the heterogeneity-induced circulations and can also dissipate the heterogeneity (Wang et al. 2011). By maintaining clouds at the same position (no movement due to advection), length scales can be calculated without the need for advanced cloud tracking. Additionally, the cloud shading effect is more isolated and therefore easier to analyze. To explore the potential extent of our cloud shading results for less idealized conditions, a simulation with a weak background flow is added and briefly discussed.

The surface conditions are based on those of Vilà-Guerau de Arellano et al. (2011). For the soil moisture content $\left(\Phi_{w}\right)$, a value of 0.218 is chosen for all levels because this represents Amazonian soil moisture conditions during the dry season, during which the TROFFEE campaign took place. Amazonian soil moisture fraction varies between 0.2 and 0.35 , depending on season and location (Camargo and Kapos 1995). For $T_{\text {soil }}$ a temperature of $300 \mathrm{~K}$ is chosen. The roughness lengths $z_{0}$ and $z_{0 h}$ (momentum and heat) are set to $0.035 \mathrm{~m}$. The simulations start just before sunrise at 0620 LT (local time; UTC -4) and run for $10 \mathrm{~h}$. To analyze the effect of cloud-induced surface heterogeneity, four experiments were set up (Table 1).

The first experiment (SHA) is a case where shallow $\mathrm{Cu}$ forms with cloud shading enabled. The DALES4 model is expanded to take into account the effect of shading on the incoming shortwave radiation by calculating the transmissivity of the clouds, $t_{\mathrm{c}}$, using

$$
t_{\mathrm{c}}=\frac{5-\mathrm{e}^{-\tau}}{4+3 \tau(1-f)} .
$$

Table 1 Experimental set-up for the experiments using the DALES model

\begin{tabular}{ll}
\hline Experiment & Important properties \\
\hline Shaded (SHA) & $\begin{array}{c}\text { Shading depends on optical depth of } \\
\text { clouds } \\
\text { Radiatively transparent clouds }\end{array}$ \\
Transparent (TRA) & $\begin{array}{l}\text { Cloud formation disabled } \\
\text { No clouds (NOC) }\end{array}$ \\
Average surface flux (AFL) & $\begin{array}{c}\text { Uniform prescribed fluxes equal to } \\
\text { the SHA domain average }\end{array}$ \\
\hline
\end{tabular}


Fig. 1 Shading (left) and transparent (right) clouds are presented. Here black surface represents shaded area, while unshaded areas are represented by grey surface. Straight arrows indicate the motion of heat and moisture, while wiggly lines represent incoming radiation, which is reduced below shading cloud. The length scales $\lambda_{\tau}, \Lambda_{\tau}$, $\lambda_{w}$, and $\Lambda_{w}$ respectively represent the length scale of the clouds, the inter-cloud distance, the length scale of the thermals and the inter-thermal distance

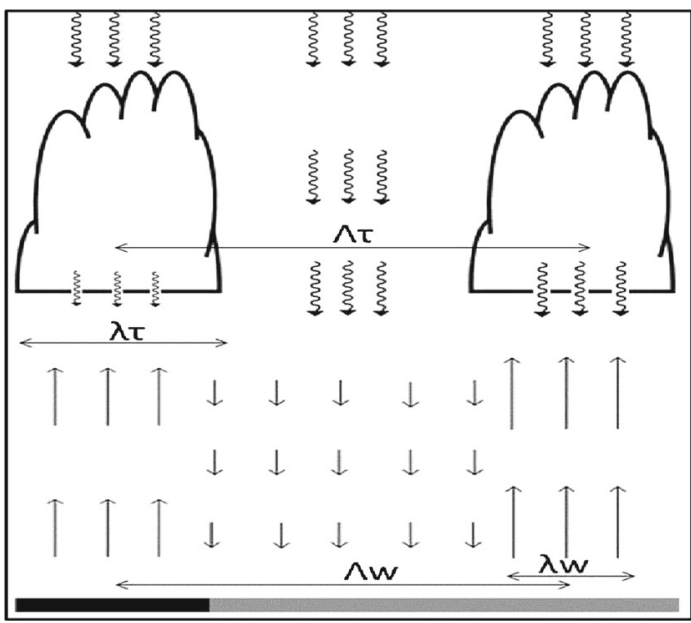

This method is similar to the shallow Cu shading introduced by Vilà-Guerau de Arellano et al. (2005), based on Joseph et al. (1976). In Eq. 1, $f$ represents the scattering-phase functionasymmetry factor, equal to 0.86 for shallow $\mathrm{Cu}$ (Joseph et al. 1976), and $\tau$ is the optical cloud depth calculated according to Stephens (1978) as

$$
\tau=\frac{3}{2 \rho_{\mathrm{l}}} \frac{W}{r_{\mathrm{e}}},
$$

where $W$ is the vertically integrated liquid water $\left(\mathrm{kg} \mathrm{m}^{-2}\right), r_{\mathrm{e}}$ is the effective droplet radius and $\rho_{\mathrm{l}}$ is the density of water. By applying this calculation, $\tau$ gives a two-dimensional (2D) surface projection of cloud depth. This is a widely used method for calculating $\tau$ (e.g. Slingo 1990; Vilà-Guerau de Arellano et al. 2005, 2014; Brenguier et al. 2011; Lohou and Patton 2014). For a tropical shallow $\mathrm{Cu}$ case, as studied here, observational evidence shows that $r_{\mathrm{e}}=10^{-5} \mathrm{~m}$ is an acceptable approximation of the modal droplet radius (McFarlane and Grabowski 2007; Brenguier et al. 2011). This surface shading introduces two effects: firstly a domain-averaged decrease in surface fluxes, and secondly a surface heterogeneity because the actual decreases in surface fluxes are local. The direct impact of shallow $\mathrm{Cu}$ shading on the surface is addressed in Sect. 3.1.

The second experiment (TRA) is characterized by transparent clouds. To simulate the effect of transparent clouds, the two aforementioned equations are simply ignored, resulting in a transmissivity of 1 . This experiment is used to quantify the net effect of shading due to clouds by comparing the results with the SHA experiment. Figure 1 conceptualizes this difference in transmittance of incoming shortwave radiation between the first and second experiments.

The third experiment (NOC) has cloud formation disabled (see Sect. 2.1), as our purpose is to quantify the dynamic effects of shallow $\mathrm{Cu}$ by comparing this experiment with the first and second experiments where shallow $\mathrm{Cu}$ is present. In this experiment the mass flux is zero and there is no surface shading. By performing these three experiments, we aim to systematically differentiate effects due to shading and dynamic cloud effects associated with the mass flux.

The fourth and last experiment (AFL) is one with clouds, where the sensible and latent heat fluxes are prescribed. These time-dependent fluxes are uniformly prescribed (homogenized) as the domain-averaged sensible and latent heat fluxes of the SHA experiment (see Fig. 5a), 
removing the cloud-induced dynamic heterogeneity. This experiment is in line with an earlier study by Seifert and Heus (2013), who concluded that such a homogenization does not suppress the organization of turbulence. By comparing the results between the AFL and SHA experiments, we can discriminate between the effects in the SHA experiment that are caused by a global decrease in energy due to shading, and the effects caused by a spatial variance of fluxes due to local shading of clouds. The experimental set-up deals with an idealized case with the sun directly overhead and no wind, so that clouds and shading are stationary. To study the sensitivity of the shading effect under less idealized circumstances, an additional analysis is performed, prescribing a mean geostrophic wind of $1 \mathrm{~m} \mathrm{~s}^{-1}$. This geostrophic wind is applied to experiments that in other respects are identical to SHA, TRA and AFL. To assess the potential effect of this condition, only the significant changes with respect to the non-wind experiments are discussed in Sect. 4.2.

\subsection{Analysis of the DALES Model Results}

The main focus of this research is the length scales associated with thermals and the circulations generated in the ABL (see Sect. 1). The length scales of interest are the horizontal length scale of the circulation structures $\left(\Lambda_{w}\right)$, the horizontal size of the clouds $\left(\lambda_{\tau}\right)$, which are closely related to the horizontal size of the thermals $\left(\lambda_{w}\right)$, and the distance between clouds $\left(\Lambda_{\tau}\right)$. Here $\lambda_{w}$ and $\Lambda_{w}$ are both spatial scales of turbulence, where the first describes the width of thermal updrafts and the second describes the inter-thermal distance associated with these updrafts. These length scales are conceptualized in Fig. 1, and their calculation is based on two methods: autocorrelation and spectral analysis. The thermal scales are calculated based on the vertical velocity and evaluated at $1000 \mathrm{~m}$ height, which is approximately the middle of the mixed layer at noon, while the cloud scales are calculated based on $\tau$.

\subsubsection{Autocorrelation}

Several methods exist to calculate the length and time scales of the clouds $\left(\lambda_{\tau}, T_{\tau}\right)$ and thermals $\left(\lambda_{w}, T_{w}\right)$. One of the approaches to determine time scales is cloud tracking (Jiang et al. 2006; Dawe and Austin 2012; Heus et al. 2013), which proved to be successful in analyzing time scales. However, a less computationally intensive autocorrelation function is also able to calculate these time scales and has been successfully applied to determine the relevant length scales of turbulent dispersion under different ABL conditions (e.g. Lenschow and Stankov 1986; Dosio et al. 2005; Verzijlbergh et al. 2009). For our study an autocorrelation function is used, based on Taylor (1921) and Stull (1988). To calculate the length and time scales, the calculated autocorrelation function $(\rho)$ (e.g. Hinze 1975; Dosio et al. 2005) is given by

$$
\begin{aligned}
& T=\int_{0}^{\rho^{t}=0} \rho^{t}(t) \mathrm{d} t, \\
& \lambda=\int_{0}^{\rho^{L}=0} \rho^{L}(L) \mathrm{d} L,
\end{aligned}
$$

where $\rho^{L}$ and $\rho^{t}$ represent the autocorrelation in space and time, respectively, with $L$ and $t$ representing the space and time shift, respectively. Since the autocorrelations oscillate around zero for longer length/time scales, we set the upper integration limit to the value where the autocorrelation first becomes zero. These functions will be applied to the vertical velocity $w$ and the optical depth $\tau$. The autocorrelation is applied at all $x, y$ locations for $\tau$ and for $w$ 

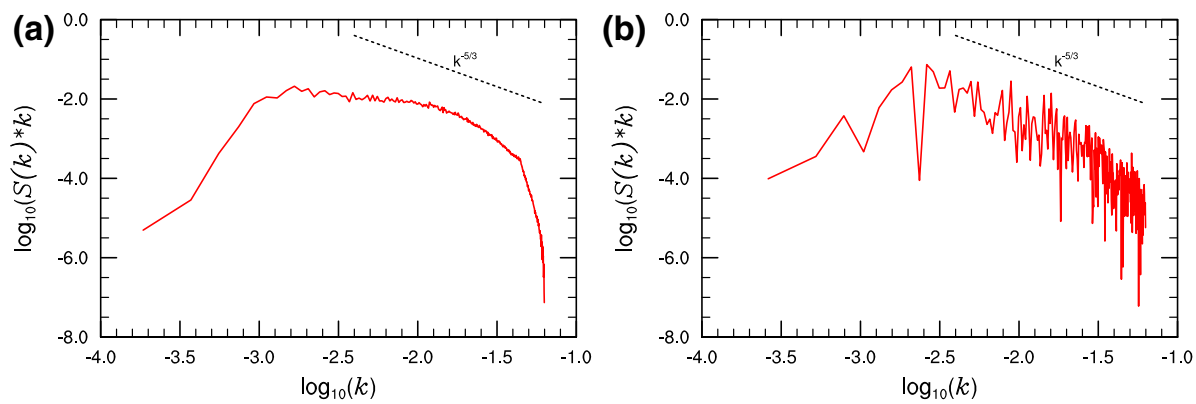

Fig. 2 Example of the spectral density $S_{w}(k)$ of a a 2D spectral analysis, and $\mathbf{b}$ a $1 \mathrm{D}$ spectral analysis. The dotted black line represents a slope of $k^{-5 / 3}$

at all $x, y$ locations at 100, 300, 500, 800, 1000, 1300, 1500, 2000 and $2400 \mathrm{~m}$, in order to cover the entire sub-cloud layer during the simulations.

\subsubsection{Spectral Analysis}

In contrast to the autocorrelation function, which quantifies the range over which a variable is correlated, spectral analysis provides length scales at which similar signals occur. This is therefore a useful method to calculate the length scales of coherent circulation structures $\left(\Lambda_{w}\right)$ and inter-cloud distance $\left(\Lambda_{\tau}\right)$. Previous studies with LES have frequently used 1D (Dosio et al. 2005) or 2D (Hellsten and Zilitinkevich 2013; Pino et al. 2006; De Roode et al. 2004; Jonker et al. 1999) spectral analysis to analyze different ABL variables. Pino et al. (2006), for example, used 2D spectra to analyze the length-scale evolution of turbulent variables during the decay of convective turbulence under flows characterized by different shear. These studies only applied this method to clear ABL but showed that spectral analysis is a useful tool for the study of length scales of turbulent variables.

The LES data are evaluated on an equidistant grid. A comparison between 1D and 2D spectra of the same data shows that 2D spectra represent the energy cascade more accurately (Fig. 2). We therefore used a 2D discrete forward Fourier transformation to analyze the size and structure of the thermals. This $2 \mathrm{D}$ transform takes signals in both horizontal directions into account, in contrast to a 1D transform on 1D averaged data, thus making the statistical analysis more robust.

The Fourier transform $\left(F\left(k_{x}, k_{y}\right)\right)$, as a function of the wavenumbers in the $x$ and $y$ direction, is converted to the energy density $\left(E\left(k_{x}, k_{y}\right)\right.$; Eq. 5). Taking the contributions of $k_{x}$ and $k_{y}$ to the composed wavenumber into account (Eq. 6), the spectral density $S(k)$ is calculated from the energy density as the angle-integrated modulus (Eq. 7).

$$
\begin{aligned}
E\left(k_{x}, k_{y}\right) & =\left|F\left(k_{x}, k_{y}\right)\right|^{2}, \\
k & =\sqrt{k_{x}^{2}+k_{y}^{2},} \\
S(k) & =\frac{1}{\Delta k} \sum_{k^{\prime}=k-\frac{\Delta k}{2}}^{k+\frac{\Delta k}{2}} E\left(k^{\prime}\right) .
\end{aligned}
$$

Here, $\Delta k=2 \pi / \lambda$, with $\lambda$ being the domain size. As such, $\Delta k$ is equal to $\Delta k_{x}$ and $\Delta k_{y}$ is equal to the minimum wavenumber. This equation is evaluated at $k=n \frac{2 \pi}{\lambda}$ with $n=1,2, \ldots, k_{\max }$, 
where $k_{\max }$ is defined as our maximum spectral domain size $\left(\sqrt{k_{x, \max }^{2}+k_{y, \max }^{2}}\right)$. Applying the $2 \mathrm{D}$ spectra transfer to the vertical velocity $(w)$ variance results in a typical result such as that presented in Fig. 2a. It is apparent that the resulting spectrum satisfactory follows the $k^{-5 / 3}$ decay in the inertial sub-range, as supported by theory (Kolmogorov 1941). The sudden drop in the small scales in the 2D spectra occurs because the DALES model uses a finite-difference method, and thus does not explicitly resolve all energy at the smallest scales. In order to resolve this, the energy dissipation at the smallest scales is calculated by the subgrid model.

Pino et al. (2006) used these 2D spectra to analyze the length scales from LES data for the different turbulent variables. We calculate $\Lambda$ using the same method (Eq. 8), with $a=-1$, similar to Pino et al. (2006), because our interest lies in the length scales rather than the wavenumbers. By choosing this value for the exponent, we directly determine the length scales weighted for the energy spectrum.

$$
\left(\frac{1}{2 \pi} \Lambda_{\Phi}\right)^{-a}=\frac{\int_{0}^{\infty} S_{\Phi}(k) k^{a} \mathrm{~d} k}{\int_{0}^{\infty} S_{\Phi}(k) \mathrm{d} k}
$$

\section{Results and Discussion}

\subsection{Impact of Shallow Cu Shading on the Surface}

Cloud shadows are characterized by a decrease in skin temperature, as can be seen for the SHA case (Fig. 3b). Such an effect is not present in experiment TRA (Fig. 3d). The decrease in $T_{\text {skin }}$ is the cause of the instantaneous decrease in latent heat flux $(L E)$ and sensible heat flux (SH) in the shaded areas, as discussed by Vilà-Guerau de Arellano et al. (2014) and Lohou and Patton (2014). By horizontally averaging these fields at the surface, we find that there is not much difference in the spatial averaged $T_{\text {skin }}(312.1 \mathrm{~K}$ for experiment SHA versus $312.8 \mathrm{~K}$ for experiment TRA). However, cloud shading leads to a significant increase in variance of $T_{\text {skin }}$, from $0.03 \mathrm{~K}^{2}$ (TRA) to $2.26 \mathrm{~K}^{2}$ (SHA). This indicates that spatial fluctuations exist for both shading and transparent clouds, but the perturbation of incoming shortwave radiation (by shading) induces a dynamic surface heterogeneity that is much stronger than the surface heterogeneity without this perturbation. The variance in $T_{\text {skin }}$ in the experiment with transparent clouds is caused by a response of the coupled surface to the presence of clouds through the mass flux that leads to mixed-layer drying, as was shown by van Stratum et al. (2014). The mixed-layer drying effect is very small but still noticeable in the variance of $T_{\text {skin }}$. Due to the highly non-linear behaviour of Clausius-Clapeyron, a similar but more pronounced effect is found for the saturated moisture content at the surface $\left(q_{\mathrm{s}}\right)$.

Convective cell structures are present in both cases (Figs. 3, 4). Rising motions under the clouds and downward motions in clear sky indicate that the clouds are rooted on the moist thermals, as was earlier observed by LeMone and Pennell (1976) and confirmed in LES with cloud shading present by Lohou and Patton (2014). By creating differences in surface temperature, and thereby density, flow is generated from shaded patches (lower temperature) to unshaded patches (higher temperature). Note that the flow caused by dynamic heterogeneity has an opposite direction to the thermal circulation on which clouds grow. Because both cases show similar circulations and length scales, it is hard to distinguish between them. More mathematical methods are therefore needed to determine the differences and analyze the length scales. This is presented in Sects. 3.4, 3.5 and 3.6 via a length-scale analysis. 

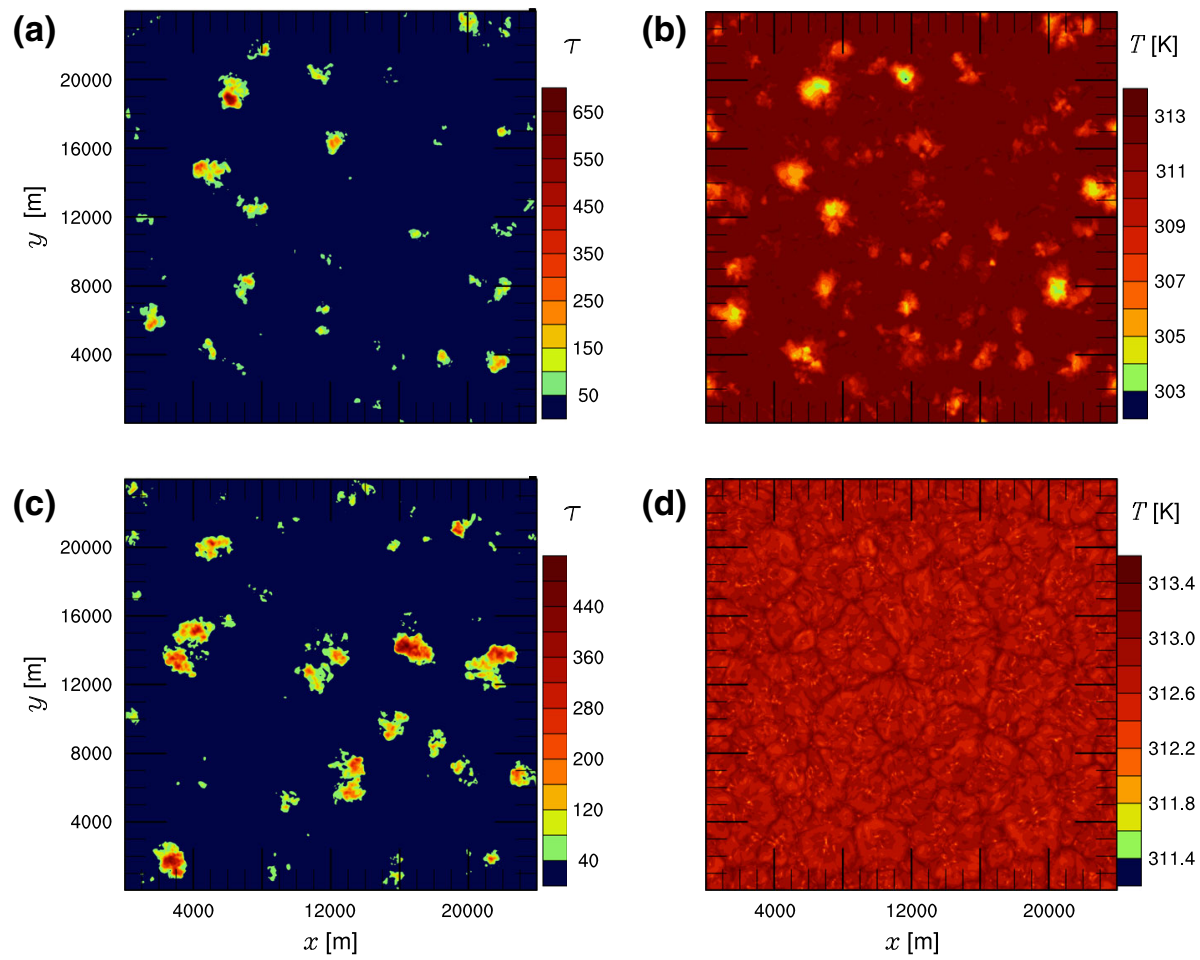

Fig. 3 Instantaneous horizontal cross-section of $\mathbf{a}, \mathbf{c}$ optical depth $(\tau)$ and $\mathbf{b}, \mathbf{d}$ skin temperature $\left(T_{\text {skin }}\right)$. The upper panels a, b refer to the SHA experiment, while the lower panels $\mathbf{c}, \mathbf{d}$ refer to the TRA experiment. The horizontal cross-sections are taken at 1400 LT, which is the time of maximum cloud activity (see Fig. 7)
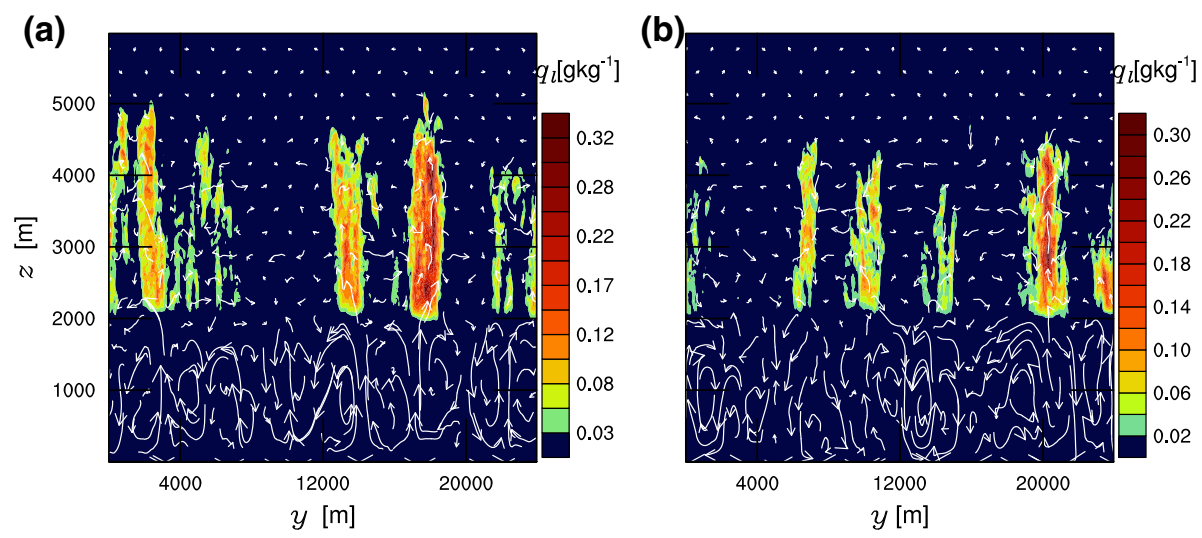

Fig. 4 Instantaneous vertical cross-section of $\left(q_{1}\right)$ at $x=100 \mathrm{~m}$ with the vectors representing the composite wind components of $v$ and $w$. a Depicts the SHA experiment and $\mathbf{b}$ the TRA experiment. The vertical crosssections show the average over the period 1300-1400 LT 

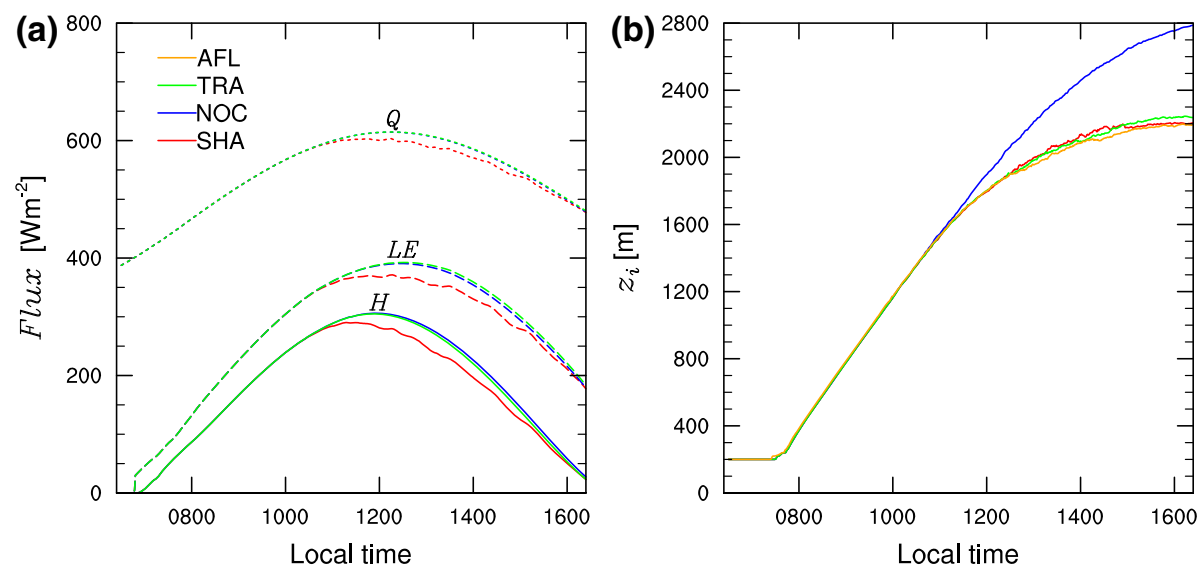

Fig. 5 Evolution of: a the domain-averaged latent heat flux ( $L E$ dashed), the domain-averaged sensible heat flux ( $H$ solid) and the domain-averaged net radiation $(Q$ dotted $)$ and $\mathbf{b}$ the evolution of the mixed-layer height, $z_{i}$, which is the domain-averaged mixed-layer height defined as the altitude at which the virtual potential temperature gradient exceeds $50 \%$ of its maximum value (Ouwersloot et al. 2011)

\subsection{Impact on Boundary-Layer Characteristics}

The local drop in $T_{\text {skin }}$ (due to shading) influences the surface energy balance (Fig. 5a). We find that the introduction of cloud dynamics in the TRA experiment has only a minor effect on the net radiation and surface fluxes, as shown by the small difference between experiments TRA and NOC. Both runs display sinusoidal shapes driven by the incoming shortwave radiation. The introduction of shallow $\mathrm{Cu}$ shading on the surface in experiment SHA lowers the surface energy balance, as is shown by lower surface energy fluxes compared with experiments TRA and NOC and a stronger deviation from the sinusoidal behaviour shown in Fig. 5a. This decrease due to cloud shading was also observed by van Kesteren et al. (2013) and was further corroborated by the LES analyses of Vilà-Guerau de Arellano et al. (2014) and Lohou and Patton (2014). Differences in behaviour between the cases indicate that the introduction of cloud venting in experiments SHA and TRA does not have a major effect on average surface fluxes, but that the variability in the SHA experiment is mainly due to the surface shading of those clouds.

A larger effect of cloud presence is visible in the growth of the mixed layer (Fig. 5b). The mixed-layer height is defined by the location where the vertical gradient of $\theta_{\mathrm{v}}$ reaches $50 \%$ of its maximum value (Ouwersloot et al. 2011). For the cloudy cases, SHA and TRA, this height is equivalent to the height of the sub-cloud layer. Here the mixed layer of the three cases starts to deepen at around $0800 \mathrm{LT}$, and has the same evolution until approximately 1030 LT, which is when clouds start to form in experiments TRA and SHA (see Fig. 7). Cases TRA and SHA introduce cloud venting (Neggers et al. 2006; van Stratum et al. 2014), which induces transport out of the mixed layer, thereby hampering mixed-layer growth, as is indicated by the higher mixed-layer heights after 1030 LT for the NOC experiment. The reduction in surface fluxes and the surface heterogeneity induced by AFL and SHA do not have a major effect on mixed-layer growth, as is shown by the similar development in experiments TRA, AFL and SHA. In conclusion, while local surface fluxes are mainly affected by the shading of solar radiation by the clouds, mixed-layer growth is primarily affected by cloud dynamics. 

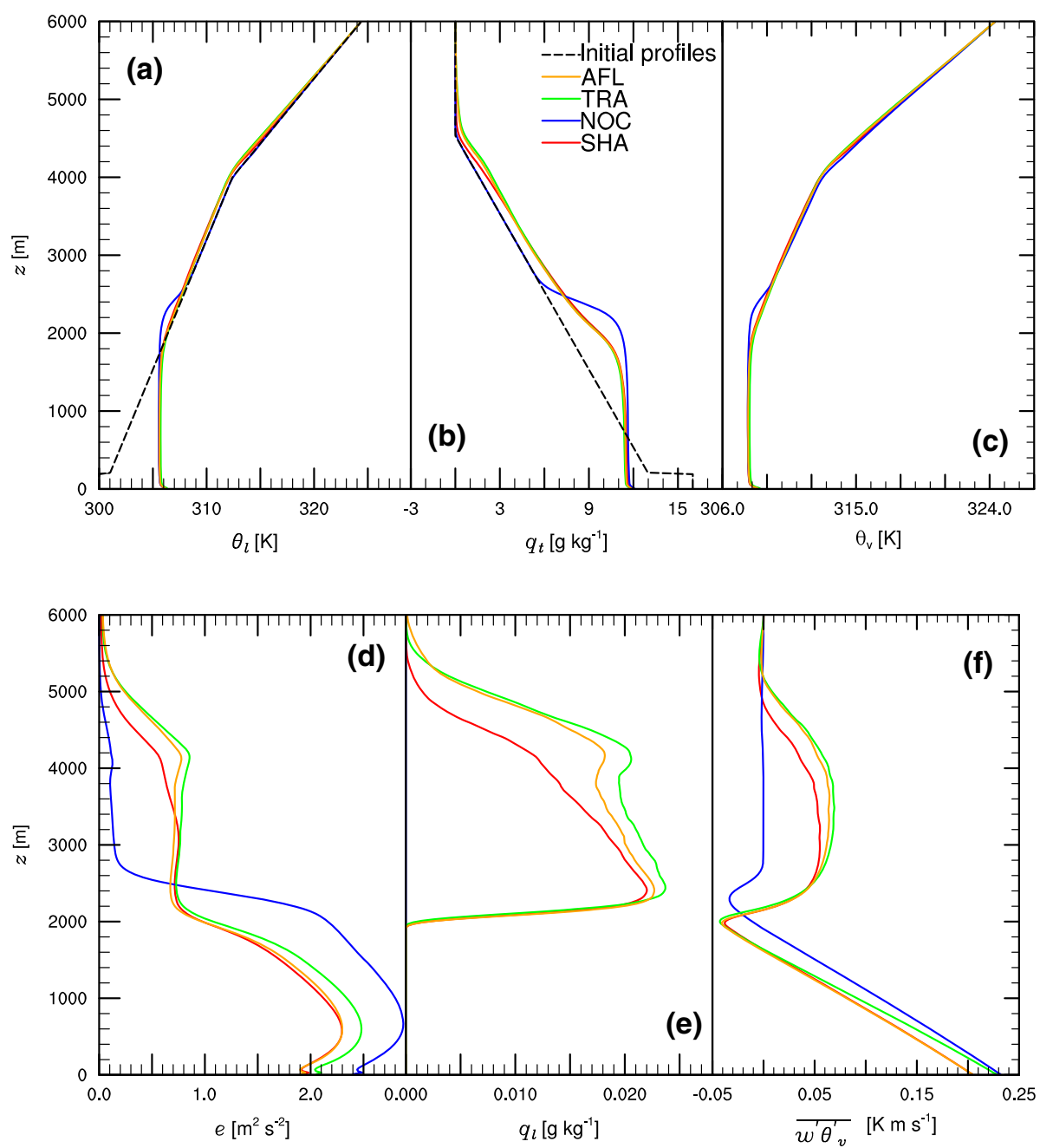

Fig. 6 Horizontally-averaged vertical profiles of a liquid potential temperature $\left(\theta_{1}\right)$, b total specific humidity $\left(q_{\mathrm{t}}\right)$, c virtual potential temperature $\left(\theta_{\mathrm{V}}\right), \mathbf{d} \operatorname{TKE}(e)$, e liquid water mixing ratio $\left(q_{1}\right)$ and $\mathbf{f}$ buoyancy flux $\left.\overline{\left(w^{\prime} \theta_{\mathrm{V}}^{\prime}\right.}\right)$. These variables are averaged for the time period 1300-1400 LT. The initial profiles of temperature and moisture are indicated by dashed black lines

The horizontally- and temporally-averaged vertical profiles of $q_{1}$, turbulent kinetic energy (TKE) and $\overline{w^{\prime} \theta_{\mathrm{v}}^{\prime}}$ are more sensitive to shallow $\mathrm{Cu}$ shading than the other three variables presented in Fig. 6. These typical ABL characteristics are averaged for the time 1300-1400 LT, which is the period of maximum cloud activity (see Fig. 7). The moisture and temperature profiles show a behaviour similar to that of the typical shallow $\mathrm{Cu}$ profiles presented by the LES studies of Siebesma et al. (2003) and Brown (2002). The $q_{\mathrm{t}}$ profile for the NOC case follows the initial profile above the inversion. This indicates that no moisture transport out of the mixed layer takes place, in contrast to the other two cases. The TRA case has a lower specific moisture content in the mixed layer than the SHA case, but a higher content in the cloud layer, which points to more moisture transport. This is supported by $q_{1}$, where it is clear 

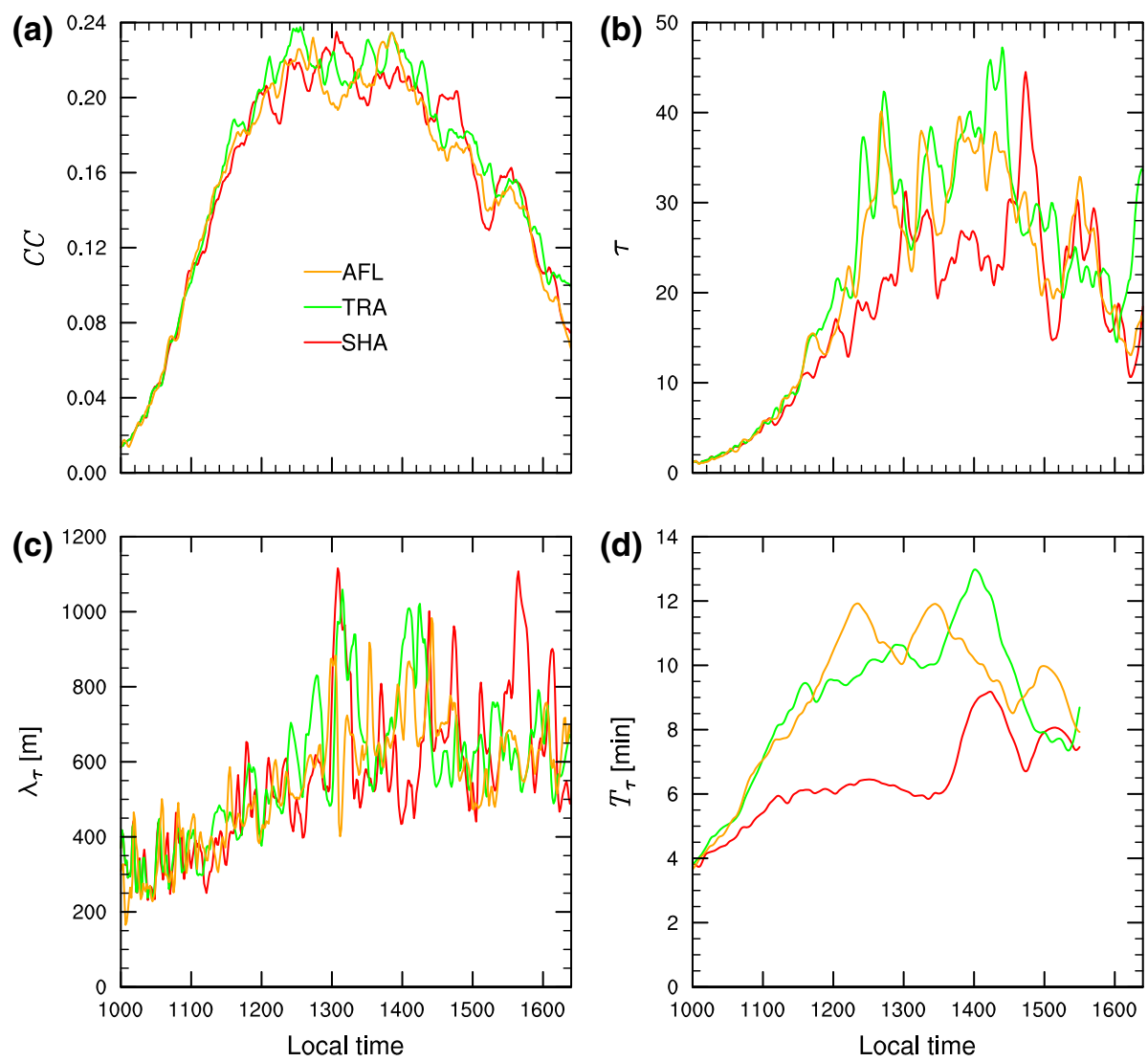

Fig. 7 The evolution of: a domain-averaged cloud cover, $\mathbf{b}$ conditional $(\tau>0)$ domain average of $\tau$, $\mathbf{c}$ length scale of shallow $\mathrm{Cu}\left(\lambda_{\tau}\right)$ and $\mathbf{d}$ time scale of shallow $\mathrm{Cu}\left(T_{\tau}\right)$

that the TRA case has more liquid water and a larger vertical extent. Our AFL case shows values for $q_{1}$ that lie somewhere between those of experiments TRA and SHA, but are closer to the TRA case. This indicates that the reduction in experiment SHA is caused not only by the domain-averaged reduction in surface energy (depicted by the AFL case), but that the local spatial partitioning of the surface fluxes plays a more important role.

In the absence of clouds, the TKE is greater in the mixed layer because turbulence is consumed to induce mixed-layer growth. Moreover, clouds in the TRA and SHA cases transport part of this energy from the mixed layer to the cloud layer, as can be seen in Fig. 6d. In the sub-cloud layer, the SHA experiment has lower TKE values than experiment TRA and is similar to the AFL case, which indicates that the reduction of TKE in the sub-cloud layer is due to the reduction in domain-averaged incoming energy. However, in the cloud layer, the AFL experiment follows the TRA experiment, which indicates that the reduction in the cloud layer is mainly due to a different partitioning at the surface and is not attributable to lower surface energy.

Due to the formation of clouds, a positively buoyant layer develops above the mixed layer (Fig. 6f). To better compare the strength of the convection between the three cases, we utilize the convective velocity scale developed by Verzijlbergh et al. (2009) and shown in Eq. 9. 
Table 2 Statistics of the results presented in Fig. 7, for the time period 1130-1430 LT, for the experiments with shading clouds (SHA), transparent clouds (TRA) and prescribed surface fluxes (AFL), showing the mean \pm temporal standard deviation of: cloud cover (cc), the spatially-averaged $\tau$ (sampled over columns in which clouds are present), length scale of clouds $\left(\lambda_{\tau}\right)$ and time scale of clouds $T_{\tau}$

\begin{tabular}{lccc}
\hline Variable & SHA & TRA & AFL \\
\hline $\mathrm{cc}$ & $0.20 \pm 0.02$ & $0.21 \pm 0.02$ & $0.20 \pm 0.02$ \\
$\tau$ & $20.8 \pm 5.6$ & $30.3 \pm 9.6$ & $27.7 \pm 8.6$ \\
$\lambda_{\tau}(\mathrm{m})$ & $582.8 \pm 157.0$ & $664.1 \pm 178.4$ & $613.7 \pm 135.4$ \\
$T_{\tau}(\mathrm{min})$ & $6.8 \pm 1.1$ & $10.5 \pm 1.1$ & $10.5 \pm 1.0$ \\
\hline
\end{tabular}

$$
w_{*}=\left(c_{1} \frac{g}{\theta_{0}} \int_{0}^{L_{z}} \overline{w^{\prime} \theta_{\mathrm{v}}^{\prime}} \mathrm{d} z\right)^{1 / 3} .
$$

This scaling is based on Deardorff (1980) and was revised by Verzijlbergh et al. (2009) to make it applicable to an $\mathrm{ABL}$ with shallow $\mathrm{Cu}$. Here, $L_{z}$ is the vertical height over which the buoyancy flux exists, $\theta_{0}$ is the surface temperature and $c_{1}=2.5$, ensuring that an integrated linear profile up to the inversion height is consistent with the theory presented by Deardorff (1980). van Stratum et al. (2014) showed that this velocity scale scales well with the cloud core velocity and therefore gives an indication of the mass flux. By applying this formula to Fig. 6f, we see that $w_{*}^{\mathrm{TRA}}=2.94 \mathrm{~m} \mathrm{~s}^{-1}, w_{*}^{\mathrm{AFL}}=2.85 \mathrm{~m} \mathrm{~s}^{-1}, w_{*}^{\mathrm{SHA}}=2.75 \mathrm{~m} \mathrm{~s}^{-1}$ and $w_{*}^{\text {NOC }}=2.55 \mathrm{~m} \mathrm{~s}^{-1}$. Although the NOC case has larger TKE values, it has a lower convective velocity scale. This is due to the introduction of latent heat release in the cloud layer in cases where clouds are present. This latent heat release generates buoyancy, which causes higher convective velocity scales. These values demonstrate that the cloud venting strength (van Stratum et al. 2014) is lower in experiment SHA than TRA. This reduction, caused by cloud shading, can partially be explained by a reduction in domain-averaged energy, as shown by the TKE and confirmed by the convective velocity scale values of the AFL experiment, and in other respects by the aforementioned counterflow caused by the shallow-Cu-generated surface heterogeneity. In short, shading reduces the development of turbulence via a global reduction in energy and the spatial redistribution of the surface fluxes. As a result, shading hampers cloud formation.

\subsection{Impact on Cloud Characteristics}

The impact of shading on the main cloud characteristics is shown in Fig. 7. To better quantify these results, the corresponding statistics for the time period 1130-1430 LT are presented in Table 2 . This period is characterized by the maximum cloud activity and an almost constant cloud cover of \pm 0.2 (see Fig. 7a). A two-sample $t$-test (Student 1908) indicated that the differences between all runs are significant for all variables for the time period 1130-1430 LT with probability higher than $99.99 \%$. Only cloud cover between experiments AFL and SHA, and $T_{\tau}$ between experiments AFL and TRA are not significantly different.

There are different timings of the fluctuations in cloud cover for the transparent and the radiation-altering clouds, related to the randomness of the induced thermals. However, on average the effect of shading on the cloud cover is very small, although significant according to a two-sample $t$-test. This nearly constant cloud cover was also found by a LES study by Golaz et al. (2001), who concluded that differences in $H$ and $L E$ did not affect cloud cover. The optical depth values (conditional domain average of $\tau$ calculated for all grid cells with 
$\tau>0$, see Fig. 7b) are in agreement with observations by McFarlane and Grabowski (2007), who indicated that shallow $\mathrm{Cu}$ with a wide probability range around $\tau \approx 10$ occur most frequently. Shading clouds have optical depths that are $33 \%$ lower than transparent clouds (21 versus 30), which indicates that transparent clouds are denser or deeper. The AFL run shows that the decrease in optical depth is partly due to a shading-induced decrease in surface energy, but that the difference between experiments SHA and TRA is again largely due to the counterflow that is generated by dynamic surface heterogeneity. This is supported by the $q_{1}$ values in Fig. 6e mentioned in the previous section. A decrease in optical depth caused by a decrease in surface radiation due to shading was also found by Jiang and Feingold (2006) in a study of the effects of aerosol radiative scattering on the development of clouds. Here, we show that a decrease in cloud optical depth is partly caused by a fall in surface energy (AFL) and partly by the counterflow generated by the induced surface heterogeneity.

There is a difference in the average length scale of clouds, $\lambda_{\tau}$ (Fig. 7c; Table 2). This indicates that shading decreases the mean horizontal extent of the clouds from 664 to $583 \mathrm{~m}$ $(-12 \%)$. Again, this is partly caused by a fall in surface energy (AFL), but the difference is mainly due to the induced dynamic surface heterogeneity. While cloud cover remains almost constant between experiments TRA and SHA, cloud size is less when shading is enabled, which indicates that more clouds are present. We address this effect in Sect. 3.6.

The residence time of clouds, $T_{\tau}$ (Fig. $7 \mathrm{~d}$ ), shows a clearer difference. Cloud shading lowers the time scale from 10.5 to $6.8 \mathrm{~min}$, which is a reduction of $35 \%$. $T_{\tau}$ behaves much more smoothly than $\lambda_{\tau}$. The reason for this is that the time scale uses time-dependent data, thereby averaging out variability in time, while this is not the case for the calculation of length scale. The decrease in cloud occurrence time shows why cloud size is smaller in the SHA experiment. Due to their shorter lifetime, cloud growth is limited. In comparison with the AFL experiment, we can conclude that the domain-averaged reduction in surface energy caused by cloud shading is not the reason for this decrease in time scale (the time scales of experiments AFL and TRA are equal), which indicates that the decrease in cloud lifetime is mainly due to the spatial partitioning of the surface fluxes and subsequent induced counterflow. This is further analyzed in the following section, where the time scales of the thermal roots of the clouds are evaluated.

We can conclude that shading leads to smaller, shallower and less dense clouds that disappear faster, which is mainly due to the shading-related dynamic surface heterogeneity. In the academical situation of a shallow $\mathrm{Cu}$ cloud remaining in the same place (absence of wind), clouds disappear faster due to the progressive weakening of the thermal because of the lower $H$ and $L E$ fluxes.

\subsection{Thermal Sizes and Their Lifetimes}

In order to determine the response of $\mathrm{ABL}$ dynamics to surface perturbations more quantitatively, we study the length scales associated with thermals. First, we compare the time-averaged length scales $\lambda_{w}$ in the NOC case, for the period 1130-1430 LT, on a similar scale as calculated for a clear ABL by Dosio et al. (2005) using LES (Fig. 8). The length scale peak lies at \pm 0.5 times the mixed-layer height in both cases, which is also supported by numerical results from Deardorff and Willis (1985) that reproduced laboratory experiments of a convective ABL. The scales are smaller at the bottom and top of the mixed layer. This is because the vertical motions in the middle of the mixed layer consist of more highly organized areas of updrafts around subsiding areas, while the motions at the surface and near the mixed-layer top consist of more isolated discrete eddies (Dosio et al. 2005). Another reason for the decrease at the surface and top of the mixed layer is that part of the kinetic energy is 
Fig. 8 The vertical profiles of $\lambda_{w}$ for experiment NOC, averaged over 1130-1430 LT, and the vertical velocity length scale found by Dosio et al. (2005). Variables are scaled by the mixed-layer height

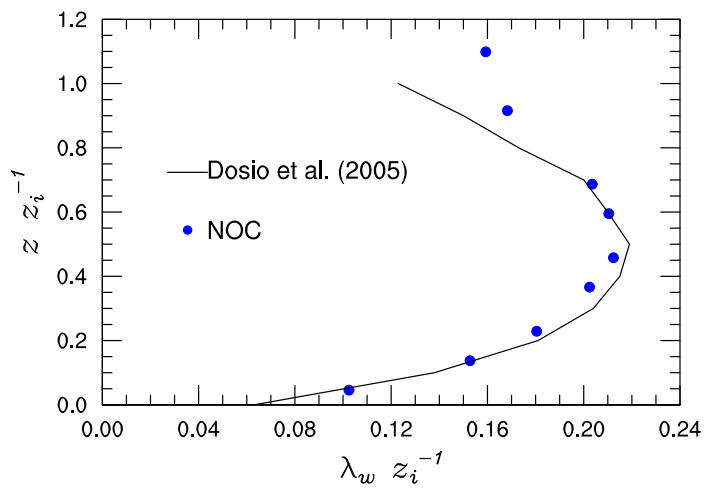

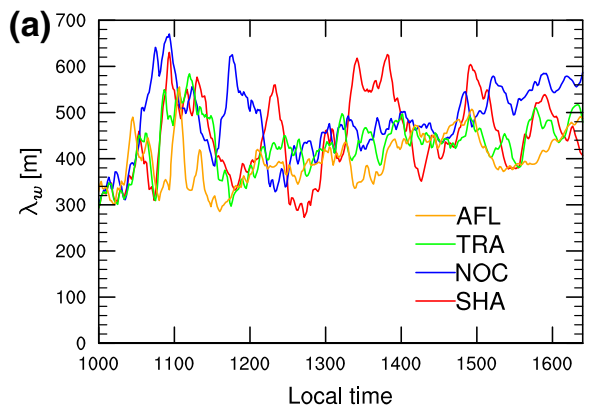
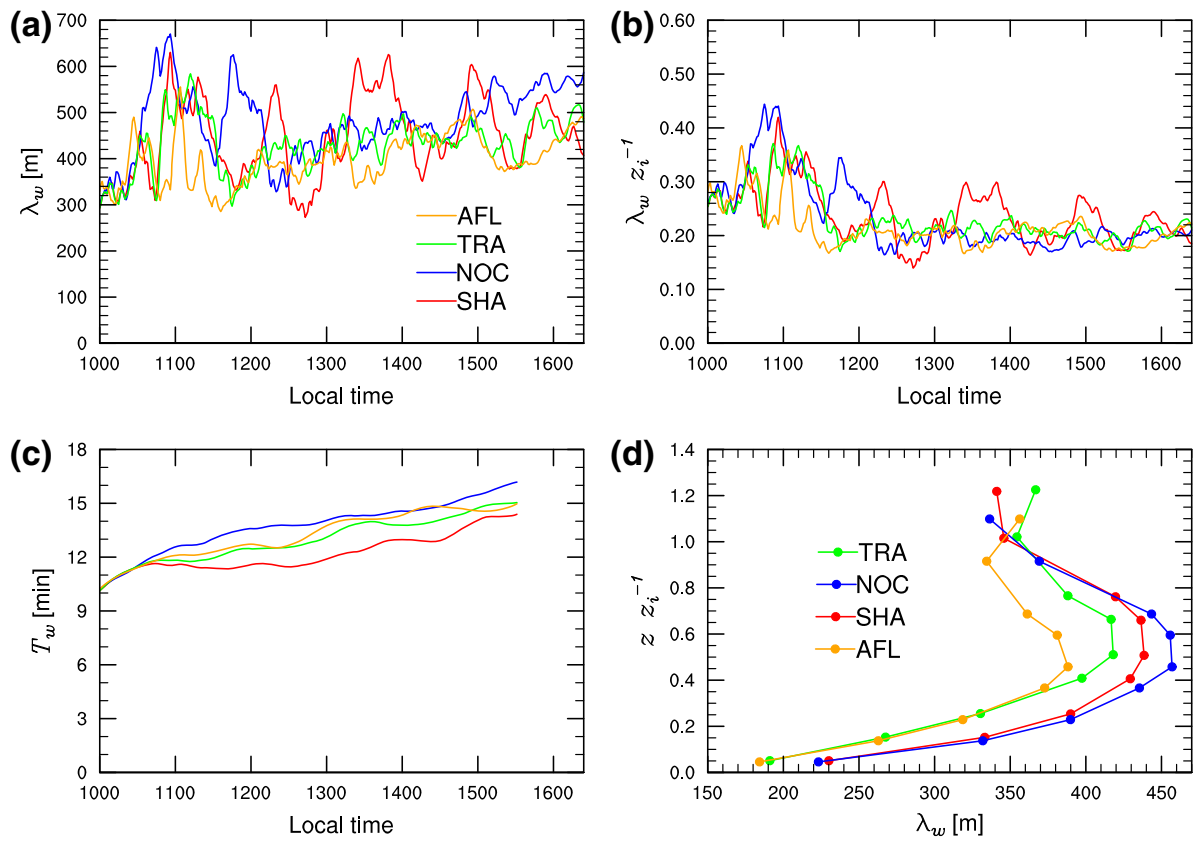

Fig. 9 The evolution of: a $\lambda_{w}$ (length scale of $w$ ) at $1000 \mathrm{~m}, \mathbf{b} \lambda_{w} / z_{i}$ at $1000 \mathrm{~m}, \mathbf{c} T_{w}$ (time scale of $w$ ) at $1000 \mathrm{~m}$ and $\mathbf{d}$ the vertical profile of $\lambda_{w}$ averaged for the time period 1130-1430 LT. The dotted lines in a indicate the average between 1130 and 1430 LT

transferred from horizontal to vertical motion and vice versa. At the top the profiles differ, but overall they have similar shapes and the values are of the same order of magnitude.

Length scales were, together with the time scale, also calculated for the other cases $\left(T_{w}\right)$ (Fig. 9a, c). According to a two-sample $t$-test, the differences between the experiments (Table 3) are all significant, with the exception of the difference between experiments TRA and NOC, normalized by mixed-layer height.

The effects of shading and cloud dynamics on thermal sizes and time scales are more difficult to distinguish between than the differences for cloud scales found in the previous section. The length scale of the shading clouds (SHA; $439 \mathrm{~m}$ ) lies somewhere between the transparent clouds (TRA) and the no-clouds experiment (NOC). This suggests that thermal size decreases due to the introduction of cloud dynamics (TRA), but that surface shading 
Table 3 Mean and standard deviation of results presented in Fig. 9

\begin{tabular}{lcccc}
\hline Variable & SHA & TRA & NOC & AFL \\
\hline$\lambda_{w}(\mathrm{~m})$ & $438.5 \pm 91.5$ & $418.1 \pm 42.0$ & $457.0 \pm 56.7$ & $388.2 \pm 42.8$ \\
$\lambda_{w} / z_{i}$ & $0.22 \pm 0.04$ & $0.21 \pm 0.02$ & $0.21 \pm 0.04$ & $0.20 \pm 0.02$ \\
$T_{w}(\min )$ & $12.1 \pm 0.6$ & $13.2 \pm 0.5$ & $14.1 \pm 0.7$ & $13.5 \pm 0.8$
\end{tabular}

The statistics are calculated between 1130 and 1430 LT for experiments SHA, TRA, NOC and AFL. Included are the mean \pm standard deviation of $\lambda_{w}$ and $T_{w}$ at $1000 \mathrm{~m}$

(SHA) partly counteracts this negative effect of cloud dynamics and increases the length scales. The length scales associated with thermals display strong variability in their evolution (Fig. 9a), as is also evident in their standard deviations. However, the differences in length scales between the runs are statistically significant and are visible not only at $1000 \mathrm{~m}$ but throughout the mixed layer (Fig. 9c). Therefore, we consider that the pattern of $\lambda_{w}^{\text {TRA }}<$ $\lambda_{w}^{\mathrm{SHD}}<\lambda_{w}^{\mathrm{NOC}}$ is consistent and that conclusions can be drawn from these results.

A major influence on the difference in length scales between experiments SHA and TRA, and NOC, is the mixed-layer height, as is shown by the normalized length scales (Fig. 9b). When normalized, the relative difference between the SHA and NOC cases decreases and the difference between experiments TRA and NOC, as shown in Fig. 9a, disappears. This indicates that the mass flux has a major impact on absolute length scales through its inhibitory effect on mixed-layer growth. The differences that can be observed between experiments TRA, SHA and AFL are diminished, but they still hold when normalized. This shows that the mixed-layer height is not the only factor that determines $\lambda_{w}$.

An effect of cloud shading (SHA) that might explain the differences between experiments SHA and TRA could be the partitioning of the surface fluxes (Fig. 5a). Due to the reduced drying of the mixed layer, caused by a lower mass flux, we observe a small increase in $L E$ and a decrease in $H$ in experiment TRA in Fig. 5, but the domain-averaged evaporative fraction (EF) remains the same between the experiments. This effect is therefore negligible and does not significantly influence the thermal formation.

Our explanation of the difference between the length scales in experiments TRA and SHA is based on strengthening and weakening effects due to shading. On the one hand (weakening effect on the strength of the thermals), in experiment SHA the shading leads to lower surface forcing and therefore a decrease in available energy (see Fig. 5a and the AFL experiment in Table 3). This causes the sensible and latent heat fluxes to diminish and leads to a decrease in TKE (Fig. 6d) and subsequent weakening of the thermals. The surface heterogeneity also induces flow from the low-temperature patches (directly beneath the clouds) to the hightemperature patches (between clouds). This flow hampers the thermal-induced circulation, thereby reducing thermal growth. On the other hand there is a strengthening effect. Due to shading, the cloud growth (in time and space) is hampered and therefore the effects of cloud dynamics are reduced, as quantified by a lower $w_{*}$ in experiment SHA than experiment TRA in Sect. 3.2. As a result, the mass flux decreases and the transport of TKE out of the mixed layer is lower, resulting in thermals growing larger. Figure $9 \mathrm{~b}$ indicates that the SHA experiment has larger normalized scales than experiment TRA, which means that the positive effect must be stronger than the negative effect. Note that, in the NOC case, the lower normalized length scale is caused by the higher mixed-layer height (Fig. 5b). The combination of lowering of surface forcing and the introduced flow by dynamic heterogeneity due to shading decreases thermal sizes compared with the TRA case. However, the shading also reduces cloud size and thereby the effect of cloud dynamics. It is clear that the reduction of the mass flux is more 
Fig. 10 The 2D spectral density $(S)$ of $w$, normalized by $k$, versus $\log _{10}(k)$. The spectra are averages for the period 1130-1430 LT. The dotted black line represents a $k^{-5 / 3}$ decay

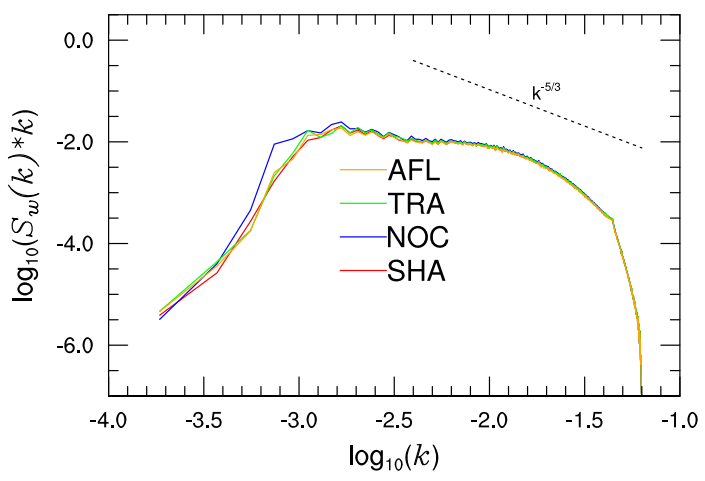

important for the length scales of the thermals than the negative effects of flux reduction and surface heterogeneity.

As Fig. 9c shows, the behaviour of the thermal time scales is much smoother than that of their length scales, similar to the difference in behaviour for the cloud scales (Sect. 3.3). Thermals do not last that long when influenced by clouds (Fig. 9b; Table 3). Taking only cloud dynamics into account (TRA versus NOC) results in a decrease of $6 \%$. Moreover, in the SHA experiment, this decrease is $14 \%$ compared with a clear ABL. This decrease in thermal lifetime is the reason for the lower $\lambda_{\tau}$ (see Fig. 7) in experiment SHA, even though the thermals are larger than in experiments TRA and AFL. This leads us to conclude that the presence of clouds (mass flux) can induce a decrease in the lifetime of a thermal by transporting TKE out of the mixed layer, and that their shading reduces their lifetime even further by lowering the surface forcing and hampering cloud growth by the cloud-induced surface heterogeneity.

\subsection{Spectral Analysis: Length Scales of Convective Structures}

The 2D spectra of $w$ (Fig. 10) correspond to a typical sub-cloud layer spectrum within which the ${\overline{w^{\prime}}}^{2}$ energy density cascade is well represented, as it follows the $k^{-5 / 3}$ decay in the inertial sub-range. There are small evident differences among the three cases. The largest deviations can be seen at the lower frequencies. The spectral peaks lie around a $\log _{10}(k)$ value of -2.8 , which corresponds to a length scale of approximately $4000 \mathrm{~m}$. The sudden drop at higher wavenumbers than $\log _{10}(k)=-1.2$ has already been explained in Sect. 2.3.2.

Based on the 2D spectra, the evolution of length scales of circulations in the mixed layer was calculated for $w$ using Eq. 8 (Fig. 11). To study the non-mixed-layer depth effects introduced in our different cases, the normalized circulation length scales (Fig. 11b) remove the effect of a deeper mixed layer. This is a logical scaling to use because thermal circulation sizes are limited by the sub-cloud-layer height (Dosio et al. 2005; De Roode et al. 2004; Pino et al. 2006), and we can therefore expect values of around 1. Statistics of these length scales for the time period 1130-1430 LT are presented in Table 4. The differences between all the scales shown in Table 4, except for $\Lambda_{w}$ for experiments TRA and AFL, are significant, with two-sample $t$-test probability above $99.99 \%$. The relative differences in length scales are large for the non-dimensionalized figure. This is primarily due to differences in the mixedlayer depth between the three cases (see Fig. 5), which determine how large the structures can grow (van Stratum et al. 2014).

De Roode et al. (2004) and Dosio et al. (2005) respectively found scales for a clear ABL around 1.3 times the mixed-layer height and 1.1-1.2 times the mixed-layer height. These 
Fig. 11 The length scales of the circulation structures $\left(\Lambda_{w}\right)$ shown in dimensional, non-dimensional and vertical dependence terms: a the evolution of $\Lambda_{w}$ at $1000 \mathrm{~m}, \mathbf{b}$ the evolution of $\Lambda_{w}$ at $1000 \mathrm{~m}$ normalized with the mixed-layer height $\left(z_{i}\right)$, $\mathbf{c}$ vertical profile of $\Lambda_{w}$ normalized with the mixed-layer height $\left(z_{i}\right)$ averaged over 1130-1430 LT
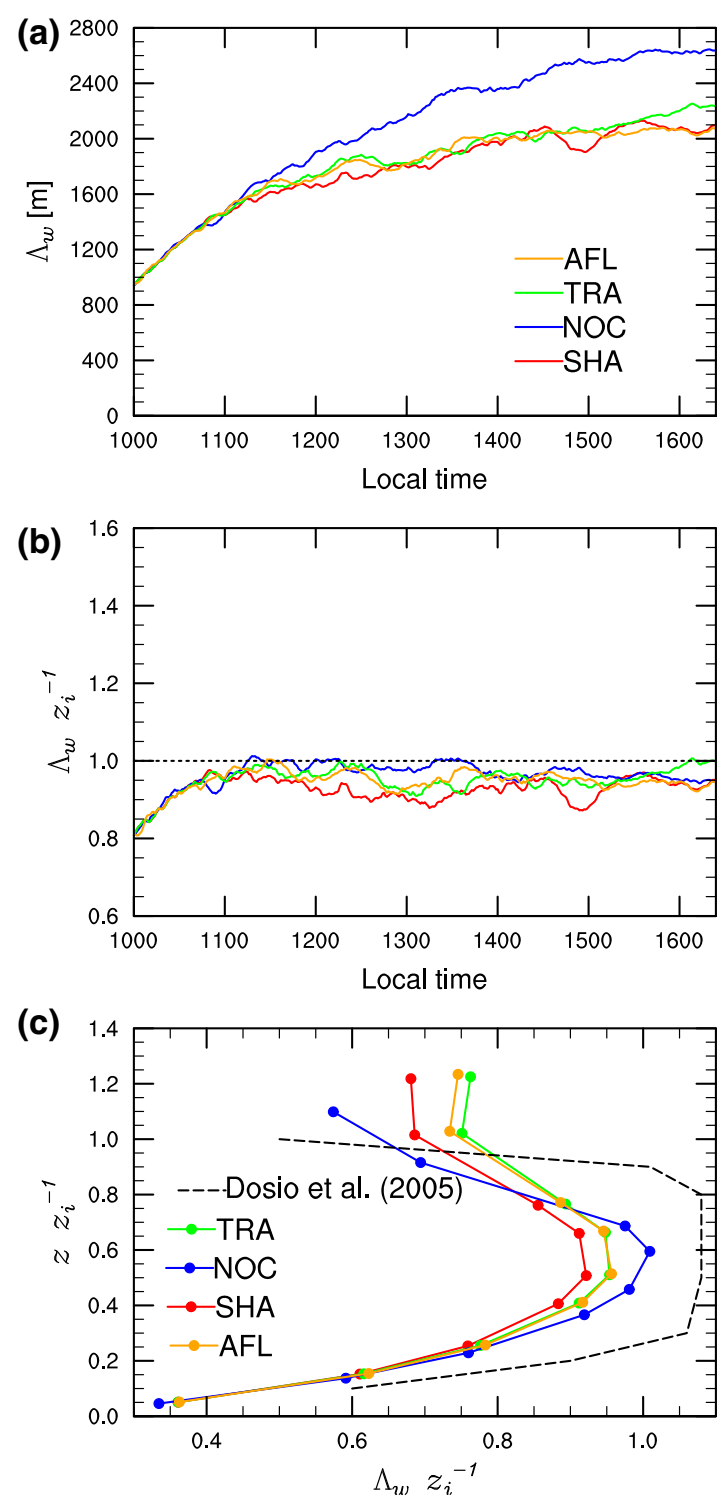

Table 4 Results presented in Fig. 11 for the experiments with shading clouds, transparent clouds, no clouds and averaged surface fluxes; mean \pm standard deviation of $\Lambda_{w}$ and $\Lambda_{w} / z_{i}$ at $1000 \mathrm{~m}$

\begin{tabular}{lcccc}
\hline Variable & SHA & TRA & NOC & AFL \\
\hline$\Lambda_{w}(\mathrm{~m})$ & $1815.8 \pm 113.7$ & $1868.3 \pm 115.9$ & $2142.8 \pm 222.2$ & $1860.2 \pm 116.8$ \\
$\Lambda_{w} / z_{i}$ & $0.92 \pm 0.02$ & $0.95 \pm 0.02$ & $0.98 \pm 0.02$ & $0.96 \pm 0.02$ \\
\hline
\end{tabular}

The standard deviations indicate the temporal standard deviations for this period 
results are comparable to the values found in this study. Here, cloud dynamics introduced in the TRA experiment have a $3 \%$ lower circulation size than experiment NOC, which comes on top of the reduced non-normalized circulation size, due to a lower mixed layer. When shading is also taken into account (SHA), the circulation size is $6 \%$ lower than in experiment NOC. This shows that, by transporting air from the mixed layer to the cloud layer, cloud dynamics (TRA) decrease the size of circulations in the mixed layer compared with a clear ABL (NOC). Introduction of the mass flux leads to transport of TKE out of the mixed layer, which reduces thermal size, which in turn reduces the strength of these circulations and thereby their size. Experiment SHA has smaller circulation sizes than experiment TRA, which is due to two factors. First, the clouds-circulation feedback is weaker, due to the shading of the surface below the clouds (a reduction in surface energy), as explained in the previous section. Secondly, the surface heterogeneity induces a counterflow that may hamper circulation strength. Table 4 shows that the AFL experiment has values similar to those of experiment TRA, which indicates that the overall decrease in energy due to shading (the first effect) is not particularly important. This leads us to conclude that the second effect, i.e. dynamic heterogeneity, is the sole determinant of the difference induced by cloud shading. This is a unique way to show that the countercirculation caused by shading-induced surface heterogeneity does indeed weaken the thermal coherence structures.

When we compare our NOC case with the clear case reported by Dosio et al. (2005) (Fig. 11c), it is clear that the normalized length scales lie within the same order of magnitude as similar vertical structure. Differences between the results of Dosio et al. (2005) and our three cases are due to different initial conditions and forcings. These profiles show that the results shown in Fig. 11a, b, concerning the differences between experiments TRA and SHA, hold throughout the whole mixed layer, while experiment NOC is larger than the other two only up to $\approx 0.7$ times the mixed-layer height. Above this height the length scales of the NOC experiment are smaller than the other two cases. This indicates that cloud dynamics (cloud venting) in experiments TRA and SHA lead to a stronger flow at higher depths in the mixed layer, resulting in a more persistent vertical velocity. This produces larger length scales for the circulation size.

\subsection{Cloud Occurrence}

Section 3.3 showed that cloud size is larger in experiment TRA with respect to experiment SHA, while cloud cover remains almost constant. Our working hypothesis is that this is caused by a compensatory increase in the number of clouds. To confirm or refute this hypothesis, the spectral density of $\tau$ was calculated for the TRA and SHA cases (Fig. 12). The SHA case has less energy than the TRA case at all frequencies, with the largest difference occurring at the lower frequencies, which would result in smaller length scales. The spectra of $\tau$ follow the $k^{-5 / 3}$ decay well at the inertial sub-range. By applying Eq. 8 to the calculated spectral densities, we calculated the associated length scales of cloud occurrence (Fig. 13a, b). The differences in length scales between the cases (Table 5) are significant according to a twosample $t$-test, except for the difference between experiments AFL and TRA for $\Lambda_{\tau}$ and $\Lambda_{\tau} / z_{i}$. The results show that the inter-cloud distance is $16 \%$ lower in the SHA experiment than in experiment TRA. Besides reducing cloud size, therefore, shading also decreases the horizontal inter-cloud distance compared with non-shading clouds. Since cloud cover remains the same, our findings indicate that shading leads to a higher proportion of smaller clouds. The decrease in inter-cloud distance is caused by a decrease in circulation size, as discussed in the previous section. Because the thermal distance decreases, the distance between the clouds, which are rooted to these thermals, also decreases. Here it is again clear that the averaged 
Fig. 12 Mean 2D spectral densities $(S)$ of $\tau$ for $1130-1430$ LT, which indicate the inter-cloud distance. The dotted black line indicates a $k^{-5 / 3}$ decay
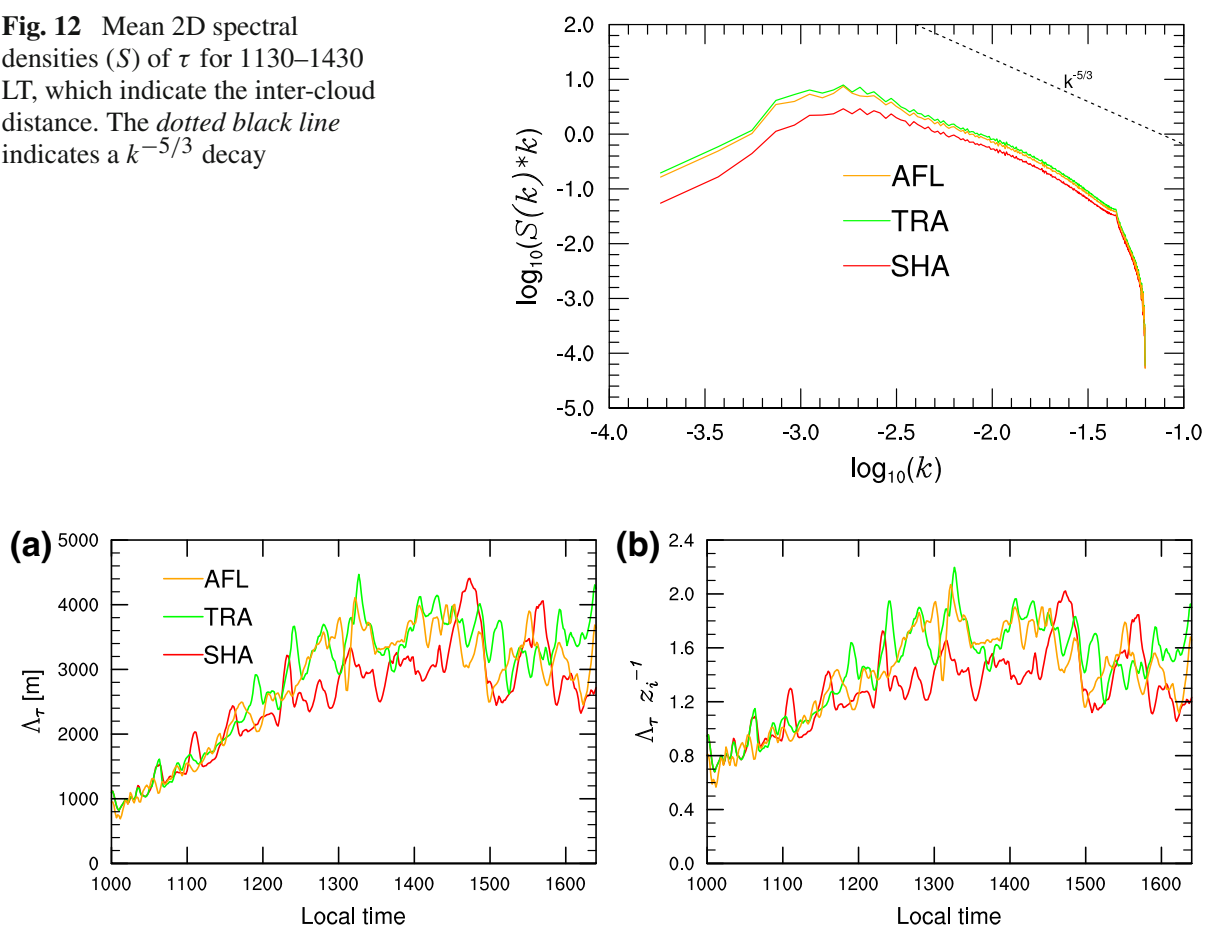

Fig. 13 Evolution of the inter-cloud distance $\left(\Lambda_{\tau}\right)$, showing: a length scale and $\mathbf{b}$ length scale normalizedby the mixed-layer height

Table 5 Statistics of results presented in Fig. 13 for experiments SHA, TRA and AFL calculated for 1130$1430 \mathrm{LT}$, showing the mean \pm standard deviation of: $\Lambda_{\tau}$ and $\Lambda_{\tau} / z_{i}$

\begin{tabular}{lccc}
\hline Variable & SHA & TRA & AFL \\
\hline$\Lambda_{\tau}(\mathrm{m})$ & $2725.4 \pm 394.7$ & $3226.2 \pm 596.6$ & $3151.2 \pm 586.4$ \\
$\Lambda_{\tau} / z_{i}$ & $1.38 \pm 0.13$ & $1.64 \pm 0.23$ & $1.61 \pm 0.22$
\end{tabular}

The standard deviations indicate the temporal standard deviations for this period

reduction in energy (AFL) plays a minor role in the effect visible in SHA. These effects must therefore be caused by the local reduction in surface energy and the corresponding generated dynamic surface heterogeneity.

\section{Discussion}

\subsection{General Discussion}

Our main findings are synthesized in a conceptual stream diagram (Fig. 14).

The physical processes that underlie this stream diagram are:

(1) Because of the mass-flux-driven shallow $\mathrm{Cu}$, momentum is transported from the mixed layer to the cloud layer, leading to a reduction in TKE and a lower mixed-layer height. 


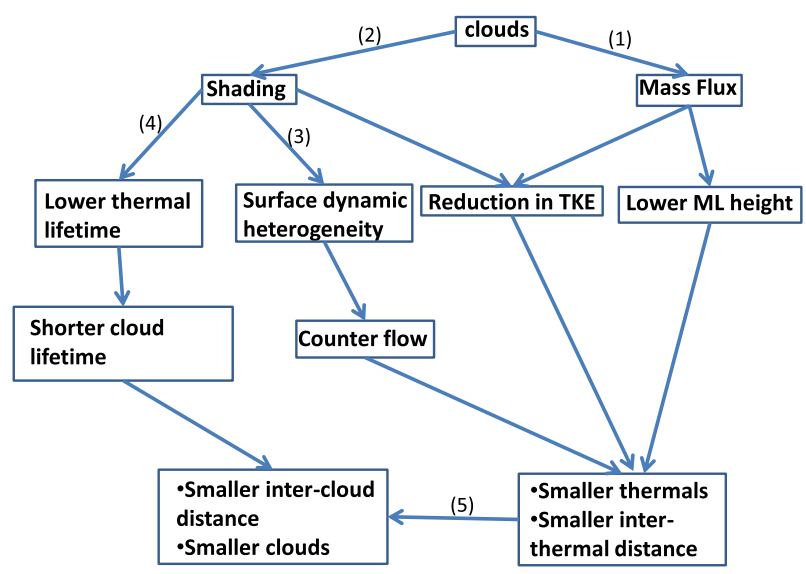

Fig. 14 Stream diagram synthesizing the results found in this paper concerning shallow $\mathrm{Cu}$ shading

The reduction in TKE yields weaker thermals, which are characterized by a smaller length scale. The reduced thermal scale is also due to a lower mixed-layer (ML) height that limits the growth of thermals. Due to the smaller thermals, inter-thermal distance decreases. This drop-off in turbulent activity is quantified by a smaller convective velocity (van Stratum et al. 2014).

(2) Shallow $\mathrm{Cu}$ produces radiative shading, which leads in turn to a reduction in incoming shortwave radiation, thereby reducing mixed-layer TKE. Due to the lower TKE (see 1), circulation cells become narrower.

(3) Shallow $\mathrm{Cu}$ cloud cover induces surface heterogeneity in incoming radiation. Due to this heterogeneity, flow is generated between patches (from colder/shaded to warmer/nonshaded). This flow is in the opposite direction to the current thermal circulation. This 'counterflow' is capable of decreasing thermal sizes by reducing circulation strength and thermal circulation sizes.

(4) Due to the radiative shading caused by clouds, shallow $\mathrm{Cu}$ reduces the energy transported to its own thermal root, thus reducing thermal lifetime. A lower thermal lifetime results in a shorter cloud lifetime and smaller clouds.

(5) Because thermals are smaller and lie closer together, inter-cloud distance and cloud size are also reduced. A reduction in inter-cloud distance compensates for the smaller size of the clouds, thereby keeping the cloud cover in balance and similar to non-shading clouds.

Surface heterogeneity is the dominant forcing mechanism having a greater effect on lowering thermal length scales than the other mechanisms. Note that generation of surface heterogeneity occurs within the boundaries of typical shallow $\mathrm{Cu}$ cloud cover. For large cloud covers (e.g. strato $\mathrm{Cu}$ ), the situation can become more homogeneous. The results (summarized in the stream diagram) show that surface shading affects length scales of the vertical velocity throughout the mixed layer. By introducing shallow $\mathrm{Cu}$ shading, we also induce a dynamic surface heterogeneity. Previous studies have shown that the influence of static heterogeneity (surface-properties-induced heterogeneity) is dependent on the length scale of this heterogeneity (e.g. Avissar and Schmidt 1998; Patton et al. 2005; Ouwersloot et al. 2011). Patton et al. (2005) showed that surface heterogeneities with length scales of 5-8 times the ABL height induce secondary circulations superimposed on the mixed-layer structure. Ouwersloot et al. (2011) suggested that static heterogeneity with length scales between 2 and 16 times 
Table 6 Statistics of the wind experiments for SHA ${ }_{W}$, TRA $A_{W}$ and AFL ${ }_{W}$, showing the mean \pm standard deviation of: $\lambda_{w}$ and $\Lambda_{w}$ at $1000 \mathrm{~m}$, and $\lambda_{\tau}$ and $\Lambda_{\tau}$

\begin{tabular}{lccc}
\hline Variable & SHAW & TRA & AFL \\
\hline$\lambda_{w}(\mathrm{~m})$ & $513.7 \pm 66.8$ & $393.9 \pm 70.1$ & $400.2 \pm 54.9$ \\
$\Lambda_{w}(\mathrm{~m})$ & $1811.3 \pm 97.7$ & $1904.7 \pm 142.9$ & $1913.9 \pm 128.3$ \\
$\lambda_{\tau}(\mathrm{m})$ & $572.6 \pm 147.3$ & $607.0 \pm 119.4$ & $610.5 \pm 123.4$ \\
$\Lambda_{\tau}(\mathrm{m})$ & $2757 \pm 483$ & $3246 \pm 747$ & $2956 \pm 407$
\end{tabular}

The standard deviations indicate the temporal standard deviation for this period

the mixed-layer height can affect the structure of the boundary layer. These studies showed that static surface heterogeneities can have a dominant effect on mixed-layer dynamics. The heterogeneity length scales we find (see Sect. 3.6) are around 1.4 times the mixed-layer height for shading clouds and are smaller than the ranges previously described. The results of the above-mentioned numerical experiments therefore indicate that our dynamic heterogeneity is too small to induce significant secondary circulations. However, our results do show that there are significant circulations generated by cloud dynamics with upward components below the clouds. These circulations are driven by clouds and eventually vanish when these disappear. The surface heterogeneity affects the length scale of these secondary circulations, but due to the shorter lifetime of the surface heterogeneity, caused by the highly dynamic behaviour of the shallow $\mathrm{Cu}$ clouds and their shading, structures are prevented from growing into superimposed secondary circulations. An interesting connection lies in the comparison between the field of aerosol dynamics and the results presented in this study. Shallow $\mathrm{Cu}$ shading tends to reduce cloud lifetimes and cloud optical depth (this study), similar to the reductive effect of smoke aerosols due to radiative scattering (Jiang and Feingold 2006; Xue and Feingold 2006).

Our findings indicate that induced dynamic heterogeneity decreases the length scales of thermals and clouds. This is related to a reduction in surface fluxes, a decrease in the time scales of these fluxes and the introduction of a counterflow due to the heterogeneity. With our cloud-induced heterogeneity we do not reproduce the dominant induced circulations treated by the aforementioned studies on fixed surface heterogeneity, but we find effects on ABL dynamics. Even though the mixed-layer averaged properties are not affected by the dynamic heterogeneity related to shallow $\mathrm{Cu}$ clouds, the distribution of thermals and the cloud properties are.

\subsection{Influence of Wind}

The introduction of a $1 \mathrm{~m} \mathrm{~s}^{-1}$ geostrophic wind into our existing free convective cases (indicated by a subscript ' $W$ ') helps us to better understand the sensitivity of our findings to less idealized conditions (Table 6 versus previous results in Tables 2, 3, 4, 5).

In all the experiments the surface fluxes are slightly larger due to the introduction of a background wind $(<1 \%)$. However, this small increase has a negligible influence on the average and local effects of surface shading and cloud dynamics. Only the experiment with shading clouds (indicated by a subscript ' $W$ ') is very sensitive to the introduction of this background wind (SHAW versus SHA). Here, the thermal size $\left(\lambda_{w}\right)$ increases significantly due to wind, while the length that characterizes the distance between thermals remains unchanged (Table 6 versus 3). During the non-wind experiments, we found that the SHA experiment is characterized by larger thermal sizes than TRA and AFL. When wind is introduced into 
those three experiments (SHAW versus TRAW and $\mathrm{AFL}_{\mathrm{W}}$ ) the difference between the cases even increases, which shows that wind $\left(\mathrm{SHA}_{W}\right)$ has an amplifying effect on the effects that are already present in the non-wind SHA experiment. The widening of the thermals in the wind case is caused by two processes introduced by wind. First, the background wind leads thermals to stretch, becoming more one-dimensionally organized than in a similar situation without wind, resulting in thermals characterized by larger length scales. Secondly, due to higher wind speeds in the mixed layer than the cloud layer, the thermal has a forward displacement. As a result, the cloud shadow only partly covers its root, thereby reducing the above-mentioned negative effect of shading and thus increasing thermal scales (Fig. 14). Besides this effect, the enhancement of local shear in the entrainment zone amplifies the increase in vertical movement in the SHAW clouds. Due to this shear effect near the entrainment zone and the shear present at the surface, more momentum is transported upward, which widens the thermals in the mixed layer. This leads to increased movements in thermals and cloud cores (see Fig. 14). These increased vertical movements for $\mathrm{SHA}_{W}$ are not large enough to cause a significant difference in widening of the SHAW clouds.

Overall, the effect of a $1 \mathrm{~m} \mathrm{~s}^{-1}$ background wind is small, and the above-mentioned shading effects under non-wind conditions are still present. On the basis of these results, we conclude that the shading effect is indeed sensitive to wind, but a $1 \mathrm{~m} \mathrm{~s}^{-1}$ background wind is too weak to result in a significant change in cloud behaviour and thermals are even strengthened. In general, these results indicate that our findings also hold for less idealized conditions, but a more complete study to analyze in depth the role of wind in the coupling between surface and clouds under realistic conditions would be useful.

\section{Conclusions}

We investigated the effects of shallow $\mathrm{Cu}$ shading and dynamics on boundary-layer characteristics by means of large-eddy simulation (LES). This was done by breaking down the complexity of the system by performing four systematic experiments with a LES model, coupled to a land-surface model. The four cases are identical in initial, soil/vegetation and free tropospheric conditions, but differ in the experimental set-up. The first experiment permits shading by the shallow $\mathrm{Cu}$, the second represents radiatively transparent shallow $\mathrm{Cu}$, the third case disables the formation of clouds and the fourth experiences a prescribed homogeneously distributed radiation equal to the domain average of the calculated net radiation in the first run. These four experiments are performed with and without a weak background wind, the latter condition enabling us to study the sensitivity under less idealized conditions. The four experiments enabled us to discriminate between the effects of cloud shading and cloud dynamics, as well as to differentiate between the effects of a uniform domain reduction of available energy and a local reduction. For our analysis, we placed special emphasis on length and time scales associated with thermals, shallow $\mathrm{Cu}$ and induced thermal circulation structures. These length scales are quantified by applying autocorrelation and 2D spectral analysis to our LES data.

In agreement with previous studies we found that the formation of shallow $\mathrm{Cu}$ leads to a mass flux that decreases the mixed-layer TKE and moisture content by transporting these quantities out of the mixed layer to the cloud layer. Our results show that the introduction of only the mass flux by shallow $\mathrm{Cu}$ (radiatively transparent clouds) leads to a reduction in length scales of thermals and thermal circulation structures compared with the same quantities in a clear ABL. Due to the presence of this mass flux, the thermals below the radiatively transparent clouds are smaller and disappear more rapidly than thermals in a clear ABL. 
Besides this first-order dynamic effect, shallow $\mathrm{Cu}$ affects the surface energy balance due to shading. To analyze this, we distinguished between the effect of a domain-averaged decrease in available energy and the effect of shading-induced heterogeneity. The introduction of cloud shading, by decreasing the incoming shortwave radiation, lowers TKE production in the mixed layer compared with transparent clouds and a clear ABL. This reduction in domain-averaged incoming shortwave radiation results in a lower surface buoyancy flux. As a result, the thermal strength decreases compared with non-shading shallow $\mathrm{Cu}$. The lowering of TKE, combined with the feedbacks introduced by transparent clouds, reduces thermal lifetime and thermal distance compared with a clear ABL as well as with non-shading clouds. However, the effect of the lower domain-averaged radiation due to shading is small compared with the impact of the localized decreases in radiation by shallow $\mathrm{Cu}$ shading, and results in dynamic surface heterogeneity, which generates a counterflow (from shaded to unshaded areas). This in turn partially offsets thermal development, resulting in an ABL with thermal circulations that are characterized by horizontally smaller cells compared with non-shading clouds and clear ABLs. As a result of the reduction in the thermal lifetime of shading clouds, the cloud occurrence time and cloud size also decrease. However, a decrease in circulation size for shading clouds also leads to a decrease in inter-cloud distance. This results in an ABL characterized by a cloud population with smaller but more clouds without affecting the cloud cover compared with radiatively transparent clouds.

Shallow $\mathrm{Cu}$ shading reduces vertical transport, quantified by the convective velocity scale, which results in a decrease in mass flux. By employing a one-dimensional radiation scheme and a case without background wind, we have created the ideal circumstances to analyze shading effects, employing maximum cloud formation and keeping these clouds exactly above their own shadow for longer. When analyzing the effects of shading under a weak background wind of $1 \mathrm{~m} \mathrm{~s}^{-1}$ we find that the above-mentioned effects of shading are still present. This shows that, even under less idealized conditions, surface shading affects boundary-layer length scales. To obtain more insight into these shading effects and find out how significant they are for less extreme and more common cases, it would be useful to apply this methodology to situations with a wider range of background winds and at mid latitudes, where the incoming radiation is lower. There, the solar angle is larger and evaporation is hampered by the lower moisture capacity of a colder atmosphere. This can be further explored in future studies.

Acknowledgments The DALES model experiments were performed using the supercomputer facilities at SURFSara. Financial support was provided by project NCF-NWO SH-060-13. We wish to express our appreciation to the two anonymous reviewers for their useful suggestions.

Open Access This article is distributed under the terms of the Creative Commons Attribution 4.0 International License (http://creativecommons.org/licenses/by/4.0/), which permits unrestricted use, distribution, and reproduction in any medium, provided you give appropriate credit to the original author(s) and the source, provide a link to the Creative Commons license, and indicate if changes were made.

\section{References}

Avissar R, Liu Y (1996) Three-dimensional numerical study of shallow convective clouds and precipitation induced by land surface forcing. J Geophys Res 101:7499-7518

Avissar R, Schmidt T (1998) An evaluation of the scale at which ground-surface heat flux patchiness affects the convective boundary layer using large-eddy simulations. J Atmos Sci 55:2666-2689

Baidya Roy S, Avissar R (2000) Scales of responce of the convective boundary layer to land-surface heterogeneity. Geophys Res Lett 27:533-536 
Bonan GB (1995) Land-atmosphere interactions for climate system models: coupling biophysical, biogeochemical, and ecosystem dynamical processes. Remote Sens Environ 51:57-73

Brenguier J, Burnet F, Geoffroy O (2011) Cloud optical thickness and liquid water path—does the $k$ coefficient vary with droplet concentrations. Atmos Chem Phys 11:9771-9786

Brown AR et al (2002) Large-eddy simulation fo the diurnal cycle of shallow cumulus convection over land. Q J R Meteorol Soc 128:1075-1093

Camargo J, Kapos V (1995) Complex edge effects on soil moisture and microclimae in central amazonia forest. J Trop Ecol 11:205-221

Dawe JT, Austin PH (2012) Statistical analysis of an les shallow cumulus cloud ensemble using a cloud tracking algorithm. Atmos Chem Phys 12:1101-1119

De Roode SR, Duynkerke PG, Jonker HJJ (2004) Large-eddy simulation: How large is large enough? J Atmos Sci 61:403-421

Deardorff J (1980) Stratocumulus-capped mixed layers derived from a three dimensional model. BoundaryLayer Meteorol 18:495-527

Deardorff JW, Willis GE (1985) Further results from a laboratory model of the convective planetary boundary layer. Boundary-Layer Meteorol 32:205-236

Dosio A, Vilà-Guerau de Arellano J, Holtslag A (2005) Relating eulerian and langrangian statistics for the turbulent dispersion in the atmospheric convective boundary layer. J Atmos Sci 62:1175-1191

Golaz J, Jiang H, Cotton W (2001) A large-eddy simulation study of cumulus clouds over land and sensitivity to soil moisture. Atmos Res 59:373-392

Hellsten A, Zilitinkevich S (2013) Role of convective structures and background turbulence in the dry convective boundary layer. Boundary-Layer Meteorol 149:323-353

Heus T, van Heerwaarden C, van der Dussen J, Ouwersloot H (2013) Overview of all namoptions in dales

Heus $\mathrm{T}$ et al (2010) Formulation of and numerical studies with the Dutch atmospheric large-eddy simulation (DALES). Geosci Model Dev 3:415-445

Hinze O (1995) Turbulence. McGraw-Hill, New York, 790 pp

Huang H, Margulis S (2013) Impact of soil moisture heterogeneity length-scale and gradients on daytime coupled land-cloudy boundary layer interactions. Hydrol Process 27:1988-2003

Jiang H, Feingold G (2006) Effect of aerosol on warm convective clouds: aerosol-cloud-surface flux feedback in a new coupled large eddy model. J Geophys Res 111:D01 202

Jiang H, Feingold G, Koren I (2009) Effect of aerosol on trade cumulus cloud morphology. J Geophys Res 114:D11 209

Jiang H, Zue H, Teller A, Feingold G, Levin Z (2006) Aerosol effects on the lifetime of shallow cumulus. Geophys Res Lett 33:L14 806

Jonker HJJ, Duynkerke PG, Cuijpers JWM (1999) Mesoscale fluctuations in scalars generated by boundary layer convection. J Atmos Sci 56:801-808

Joseph JH, Wiscombe WJ, Weinman JA (1976) The delta-Eddington approximation for radiative flux transfer. J Atmos Sci 33:2452-2459

Karl T, Guenther A, Yokelson R, Greenberg J, Potosnak M, Blake D, Artaxo P (2007) The tropical forest and fire emissions experiment: emission chemistry, and transport of biogenic volatile organic compounds in the lower atmosphere over Amazonia. J Geophys Res 112:D18 302

Kolmogorov AN (1941) On the degeneration of isotropic turbulence in an incompressible viscous fluid. Dokl Akad Nauk SSSR 31:538-541

Kuang Z, Bretherton CS (2006) A mass-flux view of a high-resolution simulation of a transition from shallow to deep cumulus convection. J Atmos Sci 63:1895-1909

LeMone MA, Pennell WT (1976) The relationship of trade wind cumulus distribution to subcloud layer fluxes and structure. Mon Weather Rev 104:524-539

Lenschow DH, Stankov BB (1986) Length scales in the convective boundary layer. J Atmos Sci 43:1198-1209

Lohou F, Patton E (2014) Land-surface and atmospheric response to shallow cumulus. J Atmos Sci 71:665-682

McFarlane S, Grabowski W (2007) Optical properties of shallow tropical cumuli derived from arm groundbased remote sensing. Geophys Res Lett 34:L06 808

Neggers RAJ, Neelin JD, Stevens B (2007) Impact mechanisms of shallow cumulus convection on tropical climate dynamics. J Clim 20:2623-2642

Neggers RAJ, Stevens B, Neelin JD (2006) A simple equilibrium model for shallow-cumulus-topped mixed layers. Theor Comput Fluid Dyn 20:305-322

Ouwersloot H, Vilà-Guerau de Arellano J, van Heerwaarden C, Ganzeveld L, Krol M, Lelieveld L (2011) Layer segregation of chemical species in a clear boundary layer over heterogeneous land surfaces. Atmos Chem Phys 11:10 681-10 704 
Ouwersloot HG, Vilà-Guerau de Arellano J, van Stratum BJH, Krol MC, Lelieveld J (2013) Quantifying the transport of sub-cloud layer reactants by shallow cumulus clouds over the Amazon. J Geophys Res 118:13041-13059

Patton E, Sullivan O, Moeng C-H (2005) The influence of idealized heterogeneity on wet and dry planetary boundary layers coupled to the land surface. J Atmos Sci 62:2078-2097

Pino D, Jonker HJJ, Vila-Guerau de Arellano J, Dosio A (2006) Role of shear and the inversion strength during sunset turbulence over land: characteristic length scales. Boundary-Layer Meteorol 121:537-556

Seifert A, Heus T (2013) Large-eddy simulation of organized precipitating trade wind cumulus clouds. Atmos Chem Phys 13:5631-5641

Seneviratne SI, Lüthi D, Litschi M, Schär C (2006) Land-atmosphere coupling and climate change in Europe. Nature 443:205-209

Siebesma AP, Jonker HJJ (2000) Anomalous scaling of cumulus cloud boundaries. Phys Rev Lett 85:214

Siebesma AP et al (2003) A large eddy simulation intercomparison study on shallow cumulus convection. J Atmos Sci 60:1201-1219

Slingo A (1990) Sensitivity of the earth's radiation budget to changes in low clouds. Nature 343:49-51

Stephens G (1978) Radiation profiles in extended water clouds. II: Parameterization schemes. J Atmos Sci $35: 2123-2132$

Stephens G (2005) Cloud feedback in the climate system: a critical review. J Clim 18:237-273

Student (1908) The probable error of a mean. Biometrika 6:1-25

Stull R (1988) An introduction to boundary layer meteorology. Springer, New York, 666 pp

Sullivan P, Moeng C-H, Stevens B, Lenschow D, Mayor S (1998) Structure of the entrainment zone capping the convective atmospheric boundary layer. J Atmos Sci 55:3042-3064

Taylor G (1921) Diffusion by continuous movement. Proc Lond Math Soc s2-20:196-212

Tiedtke M, Heckley A, Slingo J (1988) Tropical forecasting at ECMWF: the influence of physical parametrization on the mean structure of forecasts and analyses. Q J R Meteorol Soc 114:639-664

van Heerwaarden C (2006) The influence of land surface heterogeneities on the entrainment of heat. M.S. Thesis, Wageningen University and Research Centre

van Heerwaarden C (2011) Surface evaporation and water vapor transport in the convective boundary layer. Ph.D. Thesis, Wageningen University and Research Centre

van Heerwaarden C, Vilà-Guerau de Arellano J (2008) Relative humidity as an indicator for cloud formation over heterogeneous land surfaces. J Atmos Sci 65:3263-3277

van Kesteren B, Hartogensis OK, van Dinther D, Moene AF, de Bruin HAR, Holtslag AAM (2013) Measuring $\mathrm{H}_{2} \mathrm{O}$ and $\mathrm{CO}_{2}$ fluxes at field scale with scintillometry: Part II-Validation and application of 1-min flux estimates. Agric For Meteorol 178-179:88-105

van Stratum B (2011) Sub-cloud heat and moisture budgets in a transient cloud-topped convective boundary layer. M.S. Thesis, Wageningen University and Research Centre

van Stratum BJH, Vilà-Guerau de Arellano J, van Heerwaarden CC, Ouwersloot HG (2014) Subcloud-layer feedback driven by the mass flux of shallow cumulus convection over land. J Atmos Sci 71:881-895

Verzijlbergh R, Jonker H, Heus T, Vilà-Guerau de Arellano J (2009) Turbulent dispersion in cloud-topped boundary layers. Atmos Chem Phys 9:1289-1302

Vilà-Guerau de Arellano J, Kim S-W, Barth M, Patton E (2005) Transport and chemical transformations influence by shallow cumulus over land. Atmos Chem Phys 5:3219-3231

Vilà-Guerau de Arellano J, Ouwersloot HG, Baldocchi D, Jacobs C (2014) Shallow cumulus rooted in photosynthesis. Geophys Res Lett 41:1796-1802

Vilà-Guerau de Arellano J, Pattorn E, Karl T, van den Dries K, Barth M, Orlando J (2011) The role of boundary layer dynamics on the diurnal evolution of isoprene and the hydroxyl radical over tropical forests. J Geophys Res 116:D07 304

Wang C, Tian W, Parker D, Marsham J, Guo Z (2011) Properties of a simulated convective boundary layer over inhomogeneous vegetation. Q J R Meteorol Soc 137:99-117

$\mathrm{Xu}$ W, Zipser EJ (2012) Properties of deep convection in tropical continental, monsoon, and oceanic rainfall regimes. Geophys Res Lett 39:L07 802

Xue H, Feingold G (2006) Large-eddy simulations of trade wind cumuli: investigation of aerosol indirect effecs. J Atmos Sci 63:1605-1622 